\title{
Are there multiple scaling regimes in Holocene temperature records?
}

\author{
Tine Nilsen, Kristoffer Rypdal, and Hege-Beate Fredriksen \\ Department of Mathematics and Statistics, University of Troms $\varnothing$, The Arctic University of Norway, \\ Troms $\varnothing$, Norway \\ Correspondence to: Tine Nilsen (tine.nilsen@uit.no)
}

Received: 8 June 2015 - Published in Earth Syst. Dynam. Discuss.: 17 July 2015

Revised: 21 March 2016 - Accepted: 18 April 2016 - Published: 28 April 2016

\begin{abstract}
The concept of multiple scaling regimes in temperature time series is examined, with emphasis on the question whether or not a monoscaling model with one single scaling regime can be rejected from observation data from the Holocene. A model for internal variability with only one regime is simpler and allows more certain predictions on timescales of centuries when combined with existing knowledge of radiative forcing. Our analysis of spectra from stable isotope ratios from Greenland and Antarctica ice cores shows that a scale break around centennial timescales is evident for the last glacial period, but not for the Holocene. Spectra from a number of late Holocene multiproxy temperature reconstructions, and one from the entire Holocene, have also been analysed, without identifying a significant scale break. Our results indicate that a single-regime scaling climate noise, with some non-scaling fluctuations on a millennial timescale superposed, cannot be rejected as a null model for the Holocene climate. The scale break observed from the glacial time ice-core records is likely caused by the influence of Dansgaard-Oeschger events and teleconnections to the Southern Hemisphere on centennial timescales. From our analysis we conclude that the two-regime model is not sufficiently justified for the Holocene to be used for temperature prediction on centennial timescales.
\end{abstract}

\section{Introduction}

The main focus of this paper is the scaling properties in palaeotemperature records at centennial and millennial timescales. In particular we study the differences in variability between glacial and interglacial climates, and we discuss the justification of separating temperature variability on different timescales into distinct scaling regimes. The notion of "scaling" in climatic time series is based on the observation that the natural variability in the Earth's surface temperature can be modelled as a persistent stochastic process, with superposed trends and quasi-periodic modes representing variability which is not included in the noise background. The latter may be attributed to solar, volcanic, greenhouse gas and/or orbital radiative forcing, or internal climate modes such as the El Niño-Southern Oscillation (ENSO). There is a considerable body of literature suggesting that longrange memory (LRM) stochastic processes are good statistical models for de-seasonalized local and global tempera- ture records on timescales from months up to a century or more (Koscielny-Bunde et al., 1996; Rybski et al., 2006; Efstathiou et al., 2011; Rypdal et al., 2013; Østvand et al., 2014). The standard continuous-time stochastic LRM processes are the fractional Gaussian noise (fGn) and fractional Brownian motion (fBm). The latter is the cumulative integral of the former, and both are said to be scale-invariant (or scaling, or fractal), even though it is only the $\mathrm{fBm}$ process that exhibits statistical self-similarity (see Sect. 3.3). The strength of persistence, or memory, in an LRM stochastic process is described by the spectral exponent $\beta$; the power spectral density (PSD) takes a power-law form $S(f) \sim f^{-\beta}$. The fGn has $-1<\beta<1$ and stationary variance, while the $\mathrm{fBm}$ has $1<\beta<3$ and a non-stationary variance that grows in time like $\sigma^{2}(t) \sim t^{\beta-1}$. The fGn is persistent (exhibits long-range memory) if $\beta>0$, and is anti-persistent if $\beta<0$. 
Because the terms "scaling regime" and "scale break/deviation from scaling" might be ambiguous, we shall define how we use them in this paper. We shall follow the glossary of Kantelhardt (2011), where a scaling regime can be identified only if a power law is valid for scales spanning at least 1 order of magnitude, be it frequency or timescale. "Deviation from scaling" is synonymous with violation of Kantelhardt's definition. The term "break in scaling" is used to separate scaling regimes with different $\beta$, where each regime complies with Kantelhardt's definition and is valid for at least 1 order of magnitude.

Ditlevsen et al. (1996) analysed the scaling in highresolution ice-core data from Greenland. Two different overlapping time series were used to create a composite power spectrum, and from this a break in scaling was identified around centennial timescales. On timescales shorter than centennial the spectrum was flat $(|\beta| \ll 1)$, while on longer timescales a non-stationary regime with $\beta \approx 1.6$ was found. One of the time series covers $0-91 \mathrm{kyr} B P$, and the other 0 $3 \mathrm{kyr}$ BP. This procedure of combining different time series into one power spectrum is problematic since the two time series reflect different climate states with different variability. The longer time series is dominated by the glacial state, and the short series only by the Holocene data. The different variability in the two states is seen clearly by direct inspection of the data, e.g. from comparing the Holocene part of the GRIP ice core (Fig. 10a) and the last glacial period from the same ice core (Fig. 12a). The standard deviation in the Holocene time series is less than half of the glacial one, and the latter looks more bursty. The records in Figs. 10a and 12a can be associated with different stochastic processes. The Holocene record is similar to an fGn with low persistence, while the records from the last glacial period exhibit strong intermittency and are associated with a high spectral exponent, $\beta \approx 1.6$.

Pelletier (1998) estimated the power spectra and scaling exponents from a deuterium record from the Vostok ice core as well as from instrumental local data, and also created composite spectra from the records. Huybers and Curry (2006) and Lovejoy and Schertzer (2012b) have studied the scaling in multiple proxy data sets covering timescales from years to millions of years. Both report a break in scaling from fluctuations decreasing with scale $(\beta<1)$ to fluctuations increasing with scale $(h>0, \beta>1)$ on a transition timescale $\tau_{c} \sim 10^{2}$ years. The break in scaling is seen from composite spectra of palaeotemperature records based on different proxies and reconstruction techniques, where many of the records span hundreds of thousands of years. Since the Quaternary climate is characterized by numerous long glacial periods and subsequent abrupt transitions into much shorter interglacials, the spectra obtained in these papers are mostly dominated by the information from the glacial climate state. Huybers and Curry (2006) suggest that the powerlaw continuum in the spectrum of surface temperature on timescales between 1 year and a century is a result of an inverse cascade in frequency space driven by the seasonal cycle forcing. The data-analysis evidence they present for this assertion is a bicoherence spectrum of the time series which shows strong phase correlation between the seasonal cycle $\left(f_{\mathrm{s}}=1\right.$ year $)$ and pairs of frequencies $\left(f_{1}, f_{2}\right)$ satisfying the resonance condition $f_{0}=f_{1} \pm f_{2}$. This phase coherence is a result of a nonlinear interaction, but it does not imply that the low-frequency modes are driven nonlinearly by the seasonal oscillation. A low-frequency mode $f_{2}$ with phase unrelated to the seasonal cycle will beat nonlinearly with the strong seasonal oscillation and produce weak sidebands on frequencies $f_{0} \pm f_{2}$. This will produce a high bicoherence even if the nonlinear interaction has very little effect on the low-frequency mode. The non-stationary scaling regime from the century timescale and longer is proposed to be the result of a nonlinear response to the Milankovitch cycle forcing. From the composite spectra, the authors infer scaling exponents in the range $\beta=0.37-0.56$ for timescales $\tau<10^{2}$ years, and $\beta=1.29-1.64$ at longer timescales. Lovejoy and Schertzer (2012b) introduce three different scaling regimes: the "weather" regime $(\beta \approx 2$ for timescales up to 10 days), the "macroweather" regime ( $\beta \approx 0.2$ for timescales from 10 days to $10^{2}$ years), and the "climate" regime $(\beta \approx 1.4$ for timescales from $10^{2}$ years and longer). Common for these studies is that they do not make a distinction between glacial and Holocene spectra.

An alternative set of physical arguments for scaling of global temperature on scales from months to millennia has been offered by Rypdal (2012), Rypdal and Rypdal (2014), and Rypdal et al. (2015). Here, a linear power-law response to stochastic forcing yields the $\beta<1$ scaling, and the powerlaw response is interpreted from an energy balance perspective where energy is exchanged between different parts of the climate system with different heat capacities and response times. A simple two-box model consisting of the mixed layer and the deep ocean is in fact sufficient to produce a signal that reproduces the scaling characteristics observed in CMIP5 models (Geoffroy et al., 2013; Fredriksen and Rypdal, 2016). Recently, Rypdal and Rypdal (2016) demonstrated that the scaling in late Quaternary glacial climate can be described as abrupt transitions superposed on a $1 / f$-noise background, and Rypdal (2016) found early-warning signals which suggest that the Greenland stadial-interstadial transitions during the last glacial period are results of bifurcations in a multidimensional dynamical system.

In the literature we find support for our hypothesis of different scaling in glacial and interglacial climate, with the scale break at centennial timescales absent for the Holocene. Blender et al. (2006) analysed the scaling properties of a $10 \mathrm{kyr}$ long climate simulation with a general circulation model (GCM), where no scale break could be detected at centennial timescales. Roe and Steig (2004) found by using a short-range memory autoregressive (AR) model that the characteristic timescales for palaeotemperature ice-core records were significantly shorter during the Holocene than 
during the last glacial period. This study is important for our reasoning, but the idea needs to be adapted to a long-memory model.

We will analyse the scaling properties in regional and hemispheric multiproxy temperature reconstructions, and also proxy-based palaeotemperature records reconstructed from deep ice cores sampled in Greenland and Antarctica. These ice-core records are the most suitable data sets we could find for studying possible scale breaks both in the Holocene and the last glacial period, due to the high temporal resolution and long duration. We will separate the ice-core records into glacial and interglacial time series and demonstrate the fundamentally different scaling properties of these climate states, and we will analyse other temperature reconstructions for the Holocene in search of a detectable scale break.

The paper is organized as follows. In Sect. 2 we address the issues of uncertainties and limitations of proxy-based reconstructions, and the implications for the existence of separate scaling regimes are discussed. Section 3 describes the scaling analysis methods employed, and information about the data used can be found in Sect. 4. The results from the analysis are presented in Sect. 5, and discussion and conclusion follow in Sect. 6.

\section{The concept of multiple scaling regimes in the Holocene}

Lovejoy and Schertzer (2012b) identify two scaling regimes in a number of Holocene temperature records. In instrumental data the transition time $\tau_{c}$ is found to be 10-30 years, in proxy/multiproxy reconstructions it is 40-100 years, and for one of the ice-core palaeotemperature records it is approximately 2000 years. Hence, it seems difficult to identify a universal $\tau_{c}$ from the data examined in that paper. For the proxy/multiproxy reconstructions that were analysed in Lovejoy and Schertzer (2012b), the time period 1500-1979 was selected because it was common to all reconstructions and the medieval warm period was avoided. However, by starting in the Little Ice Age the series are strongly influenced by steadily increasing anthropogenic, and to a lesser extent, solar forcing. A pronounced linear trend has a strong effect on the estimate of the scaling exponent from power spectra unless the time series is linearly detrended. The same is true for the Haar fluctuation analysis, which was also applied in Lovejoy and Schertzer (2012b).

Ditlevsen et al. (1996), Pelletier (1998), Huybers and Curry (2006), and Lovejoy and Schertzer (2012b) all present composite spectra based on instrumental/reanalysis and/or proxy time series for scaling analysis. There are some problems related to this, in addition to the already mentioned aspect of combining time series from the Holocene/glacial climate state. The various data sets are representative of different degrees of spatial averaging. This will affect the shape of the spectra and the estimates of the scaling exponent, because the high-frequency variability is reduced with increasing degree of spatial averaging. For the instrumental and reanalysis data we can obtain global averages, while proxy/multiproxy time series represent local, regional, or at best hemispheric temperature. There is an important difference between composite spectra and spectra from multiproxy reconstructions. Multiproxy reconstruction methods generally take geographical weighting into account, and the aim is to obtain realistic high/low-frequency variability throughout the time period covered by the reconstruction. Most composite spectra, on the other hand, do not handle these aspects in a satisfactory manner. An example of a well-designed composite spectrum can be found in Laepple and Huybers (2014), where the proxy records have been corrected for noise, and the instrumental data used were extracted from the same location where the proxy records were sampled. No attempt was made to estimate a scaling exponent from this spectrum, but it is clear that no scale break can be observed around centennial timescales.

The presence of uncertainties and noise in proxy-based climate reconstructions is an unavoidable aspect when these types of data are used for statistical analyses. The different types of uncertainties can roughly be categorized into four groups: proxy, analytical, methodological, and dating uncertainties. A key problem is that the proxy-variable relationship is generally unknown or poorly understood, and also the evolution of this relationship through time. The proxy is considered a recorder of a climatic signal of interest, with time-dependent sensitivity and possible discontinuities. The recorder system is also affected by non-climatic effects (noise), and distinguishing the signal and noise is a challenge. Many types of proxy archives demand sophisticated tools for constructing age models, hence the dating uncertainties.

In our analysis we make no attempt to account for these and other uncertainties. We can circumvent this notoriously difficult problem because uncertainties in the data cannot invalidate our main result, which is the identification of a type I statistical error in the existing literature. A type I error is the incorrect rejection of a true null hypothesis (a false positive). In our case the null hypothesis is a scaling model with a single scaling regime. Through Monte Carlo simulation of such a model we can establish a range of power spectral densities of the reconstructed temperature records that do not falsify the null hypothesis. We shall demonstrate that the null hypothesis cannot be rejected under the assumption that there are no uncertainties in the data. Hence, large error bars on these data will only increase the range of observations that are compatible with the single-regime hypothesis.

The techniques used to estimate the scaling exponent have inherently higher uncertainties on the longest timescales, due to sparse data on these timescales. A rule of thumb is that the scaling properties for a time series of length $N$ should not be estimated on timescales longer than $N / 4$, since the un- 
certainty on timescales longer (frequencies lower) than this is too large to make meaningful estimates. Suppose, for instance, we want to establish that we have scaling in annual data on scales up to 100 years. In that case we need a series which is at least 400 years long. If we want to establish the existence of a different scaling regime on timescales longer than 100 years for a time series, we need to know with reasonable certainty the spectral estimates up to one millennium. As we will demonstrate in Sect. 5, this implies that we need annually resolved records with lengths spanning several millennia to bring the uncertainty of $\beta$ below the limit needed to reject the single-regime scaling hypothesis.

Instrumental temperature data are not included in our analysis of multiple scaling regimes because previous studies do not show pronounced breaks in the scaling after detrending to account for influences from anthropogenic warming (Rypdal et al., 2013). The series are too short to detect scale breaks at centennial timescales. However, we use instrumental data in Sect. 3 for illustration of interesting features of various techniques for scaling characterization.

\section{Methods}

In our data analysis we have used a number of tools. In general, the periodogram estimator for the power spectral density (PSD) is applied for the scaling analysis of palaeotemperature time series in our study, and is the basis for significance testing when studying potential scale breaks in the time series. We have chosen the PSD because most readers are familiar with it, and because it is fully adequate for the purpose. Wavelet scalograms are not used to estimate scaling properties but rather to visualize particular features in some of the time series. Structure functions are discussed to point out the general importance of higher-order statistics and to justify a monoscaling model (fractional Gaussian noise) for the internal temperature variability in the Holocene. The Haar fluctuation function is discussed because it is strongly advocated as the ultimate scaling analysis technique by, for example, Lovejoy and Schertzer (2012a). The detrended fluctuation analysis (DFA) is an estimation technique that is commonly used for scaling analysis of climatic records, but it will not be used in this paper because it turns out to be particularly insensitive to scale breaks on scales comparable to, or larger than, one tenth of the record length. This feature will be discussed in some detail in Sect. 3.5.

\subsection{Estimation of PSD}

The periodogram is applied as an estimator for PSD for evenly sampled time series of length $N$. The stable isotope records from Greenland and Antarctic ice cores have been linearly interpolated to obtain even sampling in time. All other records are provided with even sampling. The periodogram is defined here in terms of the discrete Fourier transform $H_{m}$ as $S\left(f_{m}\right)=(2 / N)\left|H_{m}\right|^{2}, m=1,2, \ldots, N / 2$. The sampling time is the time unit, and the frequency is measured in cycles per time unit: $f_{m}=m / N . \Delta f=1 / N$ is the frequency resolution and the smallest frequency which can be represented in the spectrum, while $f_{N / 2}=1 / 2$ is the Nyquist frequency (the highest frequency that can be resolved). The periodogram has a poor signal-to-noise ratio, but since we are interested in studying the overall shape (scaling) of the spectrum, and not the power at specific spectral peaks, this is not a problem here. By presenting the periodogram in a log$\log$ plot, the scaling exponent $\beta$ can be estimated by a linear fit to the power spectrum; $\log S(f)=-\beta \log f+c$. In the present study the periodogram is log-binned before fitting to ensure that all timescales are weighted equally (Østvand et al., 2014).

We have also considered other spectral estimators for the unevenly sampled stable isotope data from ice cores, such as the Lomb-Scargle periodogram (LSP) (Lomb, 1976; Scargle, 1982), or correlation slotting (Rehfeld et al., 2011). The main motivation for looking into different spectral methods is to compare biases in the spectra that could be wrongfully interpreted as breaks in the scaling. The papers by Rehfeld et al. (2011) demonstrate that irregularly sampled data cause various problems for all spectral techniques. Slotting can be problematic because the covariance estimators may not be positive semi-definite, and could hence give negative values in the spectrum. Interpolation leads to underestimation of the spectral power at high frequencies, while the Lomb-Scargle periodogram suffers from the opposite bias: overestimation of the spectral power at high frequencies. The skill of the LSP is, as demonstrated by Rehfeld et al. (2011), dependent on the skewness of the distribution of sampling intervals. The bias will therefore differ from data set to data set. We have tested the performance of the method on surrogate data mimicking the ice-core proxy data under study. The detailed results for this test are shown in Sect. S1.1 in the Supplement. In general the method performs well, but not much better than interpolation plus standard periodogram. Results presented in the main paper are obtained using only interpolation and the standard periodogram. Scaling analysis of the ice-core data based on the LSP is included in Sect. S1.3.

\subsection{Wavelet scalogram}

The continuous wavelet transform is the convolution between a time series $x(t)$ and the rescaled mother wavelet $\Psi(t)$ :

$W(t, \tau ; x(t), \Psi(t))=\int_{-\infty}^{\infty} x\left(t^{\prime}\right) \frac{1}{\sqrt{\tau}} \Psi^{*}\left(\frac{t^{\prime}-t}{\tau}\right) \mathrm{d} t^{\prime}$,

where the asterisk indicates a complex conjugate. The wavelet scalogram (WS) is defined as $\mid W(t), \tau)\left.\right|^{2}$ and is plotted versus time and timescale. The WS is used here as a supplementary tool to the Fourier spectra. Time segments before and after the time interval where we have data were padded with zeros, as described in Torrence and Compo (1998). The 
region in $(t, \tau)$ space affected by edge effects is the region above the white line in the upper part of the WS plot shown in, for example, Fig. 11. Due to the uneven sampling of the data in this study, linear interpolation has been performed prior to computing the WS. At each time $t$ there is a characteristic sampling period in the original time series, and hence a Nyquist period. This Nyquist period is marked as the lower white curve in the WS plots. The WS below that curve does not reflect observed variability.

We have chosen two wavelet functions as the basis for our study: the Morlet wavelet, which is complex valued, and the Mexican hat wavelet (second derivative of a Gaussian), which is real valued. The wavelet scalograms from these two wavelet functions provide different information. The Mexican hat wavelet function resolves the timing of spectral peaks precisely, while the scale resolution is poor. For the Morlet wavelet function the opposite is true.

\subsection{Structure functions and scaling function}

A plethora of estimators have been developed for computing characteristic exponents for monoscaling long-range memory (LRM) processes. They all have strengths and weaknesses, but have in common that they give nonsense if the signal is not a mono- or multi-scaling process. Common for many papers by "LRM skeptics" is the uncritical use of cookbook recipes for such estimators to data that are not scaling, for instance climatic time series dominated by a specific trend (e.g. Mann, 2011). Hence, more important than estimating a characteristic exponent is to examine the general scaling characteristics of the data. A classical and useful method is to examine the probability density functions (PDFs) of the time series as it varies on different timescales. Rather than computing and plotting the PDFs, it is more common to compute the statistical moments of order $q$ of the distribution, and then plot these moments as a function of timescale $\Delta t$. Estimators employing only secondorder statistics, like the periodogram or the Haar fluctuation employed by Lovejoy and Schertzer (2012b), are not able to distinguish between scale-invariant fluctuations and variability due to trends or oscillations. Careful application of higher-order statistics like higher-order structure functions (SFs) has this ability. This can give us the possibility to separate distinct non-scaling dynamical features from the scaling, persistent noise background. In Sect. 5.1 we shall demonstrate this usefulness on a multiproxy temperature reconstruction spanning two millennia.

Given a stochastic process $x(t)$, the moments

$S_{q}(\Delta t) \equiv E\left[\mid x(t+\Delta t)-x\left(\left.t\right|^{q}\right]\right.$

are called the SFs of the process. If the process is sampled at discrete times $t=1, \ldots, N$ the empirical moments $\hat{S}_{q}(\Delta t)=(N-\Delta t)^{-1} \sum_{i=1}^{N-\Delta t}|x(i+\Delta t)-x(i)|^{q}$ constitute estimates of the structure functions. For large $\Delta t$ (when $\Delta t$ is no longer a small fraction of $N$ ) the number of independent terms becomes small, and the statistical uncertainty of the estimate becomes large. As mentioned in Sect. 2, a useful rule is that this limits the scales we can investigate to $\Delta t<N / 4$.

Let us assume that $x(t)$ is a self-similar, Gaussian, nonstationary stochastic process, i.e. a fractional Brownian motion $(\mathrm{fBm})$. Then the structure functions defined on $x(t)$ take the form

$S_{q}(\Delta t) \equiv E\left[|x(t+\Delta t)-x(t)|^{q}\right]=\Delta t^{\zeta(q)}$,

where $\zeta(q)=h q$ is the scaling function of the self-similar (monofractal) process and $h=(\beta-1) / 2$ is the self-similarity exponent. By taking the logarithm of Eq. (3) we find the linear relationship between $\log S_{q}(\Delta t)$ and $\log \Delta t$, where $\zeta(q)$ is the constant of proportionality, and hence the SFs appear as straight lines in $\log -\log$ plots with slope $\zeta(q)$. If the SFs have the form Eq. (3) (i.e. if the SFs are straight lines in $\log -\log$ plot) so that $\zeta(q)$ is defined, but $\zeta(q)$ is not a linear function, then the process is multifractal. It follows that monofractals belong to a subclass of the class of multifractals. If the SFs are not straight, the process is neither multinor monofractal, but it may still have a bursty or intermittent appearance if the PDF of the fluctuations on the short timescales is non-Gaussian with flat tails.

If a monofractal process is stationary, it is a fractional Gaussian noise (fGn), and the SFs are constant (flat) and contain no other information than the stationarity. However, as we shall see below, the SFs may still contain some useful information about the deviation from scaling if there are trends or oscillations in the data. In order to expose the scaling properties (if any) of the process, the trick is to form the cumulative sum $y(i)=\sum_{j=1}^{i} x(j)$ and then compute the SFs from this sum. The resulting SFs take the form

$S_{q}(\Delta t) \equiv E\left[|y(t+\Delta t)-y(t)|^{q}\right]=\Delta t^{\zeta(q)}$,

where $\zeta(q)=H q$ is the scaling function. $H$ is the selfsimilarity exponent of the cumulative sum $y(t)$ and the Hurst exponent of the stationary process $x(t)$. $H$ is related to the spectral exponent $\beta$ of $x(t)$ through $H=(\beta+1) / 2$.

The usefulness of the structure-function approach is illustrated in Fig. 1. Figure 1a shows the eight structure functions corresponding to $q=1,2, \ldots, 8$ for the monthly global mean surface temperature (GMST) for the period 1880-2010 derived from the HadCRUT3 data set (Brohan et al., 2006). The SFs are far from straight in the log-log plot, as they would be if the time series were non-stationary fractal (fBm) or multifractal. But the SFs are not flat either, as they would be if the time series were an fGn. The explanation for the upward bending of the curves is obviously the strong anthropogenic temperature trend. 

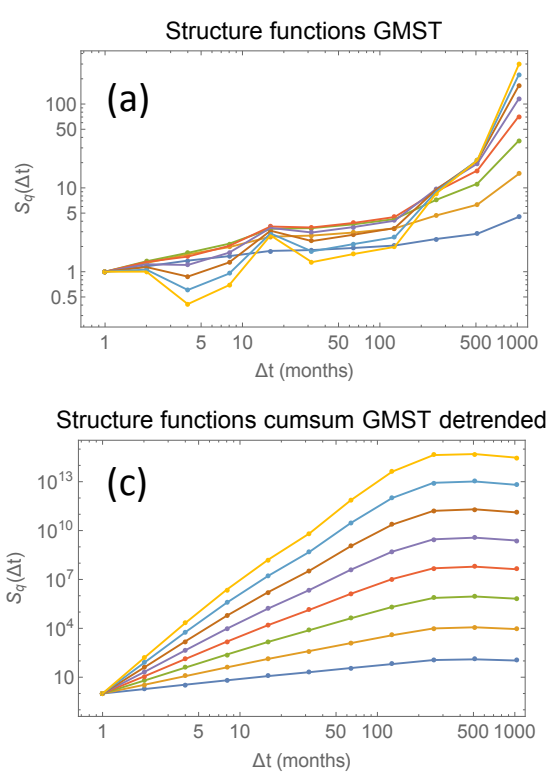

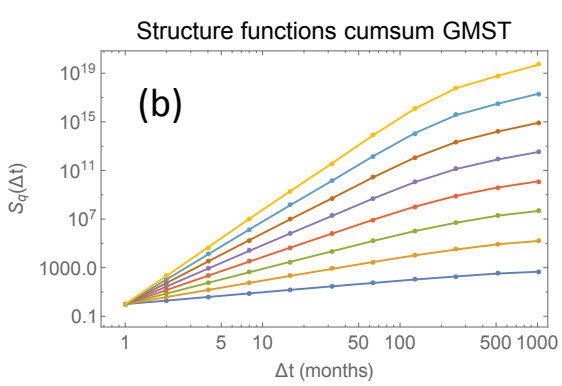

Scaling function cumsum GMST un-/detrended

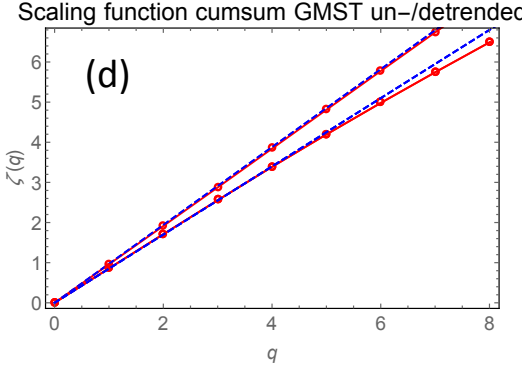

Figure 1. (a) Structure function estimates (empirical moments) $\hat{S}_{q}(\Delta t)=(N-\Delta t)^{-1} \sum_{i=1}^{N-\Delta t}\left|T\left(t_{i}+\Delta t\right)-T\left(t_{i}\right)\right|^{q}$ for the GMST (HadCRUT3) monthly record 1880-2010; $T\left(t_{i}\right) ; i=1, \ldots, N$. (b) Structure function for the cumulative sum $y_{t_{i}}=\sum_{j=1}^{i} T\left(t_{j}\right)$. (c) Structure function for the cumulative sum of the quadratically detrended GMST. (d) Scaling functions for the undetrended cumulative sum (upper line) and the detrended cumulative sum (lower line).

The underlying scaling of the noise is exposed by computing the SFs for the cumulative sum, as shown in Fig. 1 b. However, the corresponding scaling function, shown by the upper line in Fig. 1 d, has the slope $H \approx 1$, which is always the case for a signal dominated by a strong trend. The true scaling of the noise appears after a second-order polynomial fit to the record has been subtracted. The SFs for the cumulative sum of the detrended record are shown in Fig. 1c, and the corresponding scaling function by the lower line in Fig. 1 d. This line has Hurst exponent $H \approx 0.85$. The straight appearance of the scaling function tells us that the GMST is monofractal, and simple tests on the PDFs at different scales show that it is Gaussian (Rypdal and Rypdal, 2010).

The scaling functions in Fig. 1d have been computed from the slopes of the SFs in the regime of scales $\Delta t<10$ years where the SF curves are straight. The bending of these curves for large scales are due to oscillatory modes on periods around 20 and 70 years.

\subsection{The Haar fluctuation function}

A simple measure of scaling is the fluctuation analysis (FA) (for a brief review see Rypdal et al., 2013). It defines the fluctuation function $F(\Delta t)$ as the standard deviation of the data record after it has been filtered by a simple moving average with window width $\Delta t$, and hence measures the fluctuation magnitude as a function of scale $\Delta t$. The fluctuations are scaling if $F(\Delta t) \sim \Delta t^{(\beta-1) / 2}$. An issue with FA is which mean value to relate the fluctuation deviation to: the local mean in the window, or the mean of the entire data record. The latter is problematic if the fluctuations are monotonically growing with increasing scale. This problem can be circumvented by convolving the data record with the simple antisymmetric Haar wavelet (Lovejoy and Schertzer, 2012a) rather than performing the moving average. For fluctuations growing with scale it measures fluctuation differences versus scale, whereas for fluctuations decreasing with scale it measures fluctuation relative to the local mean.

The simple definition given in Lovejoy and Schertzer (2012a) starts by defining the fluctuation on scale $\Delta t$ as

$\Delta x_{t}(\Delta t)=\left|\frac{2}{\Delta t} \sum_{i=t}^{t+\Delta t / 2} x_{i}-\frac{2}{\Delta t} \sum_{i=t+\Delta t / 2}^{t+\Delta t} x_{i}\right|$

and then the Haar structure function is given by

$S_{q}^{\text {Haar }}(\Delta t)=\frac{1}{N-\Delta t} \sum_{t=1}^{N-\Delta t} \Delta x_{t}(\Delta t)^{q}$.

The Haar fluctuation function used extensively by Lovejoy and Schertzer in arguing for the existence of transitions between scaling regimes is defined as

$F^{\text {Haar }}(\Delta t)=\sqrt{S_{2}^{\text {Haar }}(\Delta t)}$.

In Fig. 2 we illustrate some features of the Haar fluctuation applied to the instrumental GMST. Figure 2a shows the 

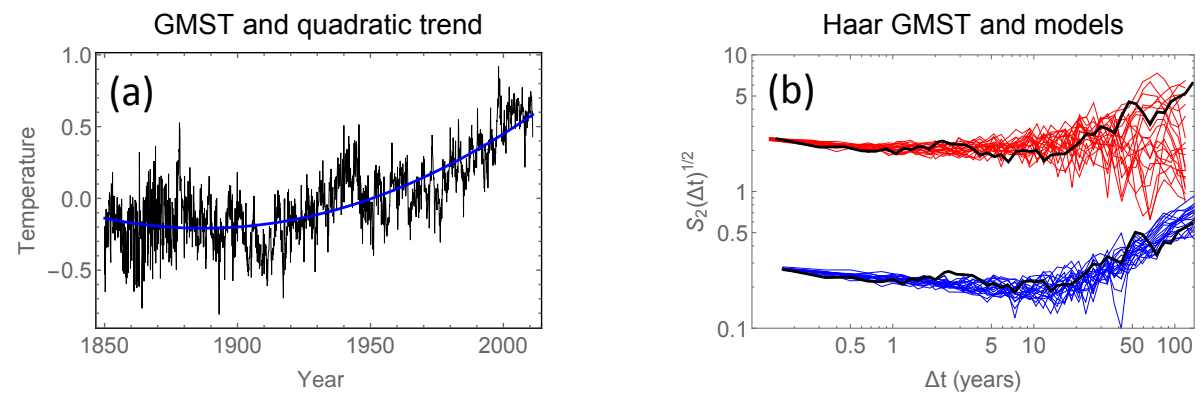

Figure 2. (a) The instrumental global mean surface temperature (GMST) 1850-2010 (black). A second-order polynomial least-squares fit to the GMST record (blue). (b) Black curves are the Haar fluctuation function of the GMST; the upper curve is multiplied by 10. The red curves are Haar fluctuation functions of 20 realizations of a model comprised of a linear combination of an fGn with $\beta=0.8$ and an fBm with $\beta=1.6$. The blue curves are the same but of a model comprised of a linear combination of an fGn with $\beta=0.8$ and the second-order polynomial trend.

GMST and a trend computed by fitting a second-order polynomial. The thick black curve in Fig. $2 b$ is the Haar fluctuation function computed from the record in a log- $\log$ plot (the upper curve is shifted by a factor of 10).

There are (at least) two different ways to model this record as a simple stochastic process. One is to assume that it is a linear combination of an $\mathrm{fGn}(\beta<1)$ and an $\mathrm{fBm}(\beta>1)$. The former will dominate the fluctuation function on the small scales, and the latter on the long scales. Hence this is a model that exhibits a scale break and two scaling regimes. We have estimated the slopes of the fluctuation function in these two regimes, and found $\beta \approx 0.8$ on the short scales, and $\beta \approx 1.6$ on the long scales. We then computed the weights of each process using the estimated variance of the GMST on the shortest and longest scales, respectively, and computed an ensemble of realizations of their linear combination. The red curves in Fig. 2 b constitute 20 realizations in such an ensemble (multiplied by a factor of 10). These curves demonstrate that the observed Haar fluctuation is consistent with this model, but also that the uncertainty in the model prediction on scales longer than a decade is so large that the observed fluctuation function here could also be consistent with a model where $\beta<1$. Another obvious way to model the record is as a linear combination of the fGn and the quadratic trend. The resulting ensemble is shown as the blue curves in Fig. 2b. The observed record is consistent with this model too, but the big difference is that in this case the model prediction on large scales is much more certain, and hence constitutes a "better" statistical model. An extra bonus is that the quadratic trend is physically well understood, since it corresponds closely to present knowledge about greenhouse forcing.

Thus, we have the choice between explaining the observation with a poor statistical model (many parameters, large prediction uncertainty, and no physics explaining the scale break) and a much better model (fewer parameters, lower uncertainty, and a clear physical explanation).
A common estimator for scaling exponents is the wavelet variance, i.e. to plot the variance of the wavelet coefficients versus scale in a log-log plot, and it is common to normalize the wavelet such that the slope for an fGn will be the spectral exponent $\beta$. In practice this can be obtained by squaring the Haar fluctuation $S_{2}^{1 / 2}(\Delta t)$ and multiplying by the scale $\Delta t$ - i.e. we compute $\Delta t S_{2}(\Delta t)$. In Fig. 3 we generate an ensemble of 10 fGn's of 1000 data points with $\beta=0.8$ and plot $S_{2}^{1 / 2}(\Delta t)$ for all realizations in the ensemble in Fig. 3a, and $\Delta t S_{2}(\Delta t)$ for the same ensemble in Fig. 3c. If we use the entire ensemble to estimate the slope we will get quite an accurate result (getting better the larger the ensemble), but if we estimate the slope from one realization we make greater errors if we include the longer timescales in the fit. This is why some authors recommend not to include longer scales than $1 / 4$ of the record length and others recommend no more than $1 / 10$. If we fit a straight line we have no reason to believe that the Haar fluctuation gives less accurate estimates than the Haar wavelet variance. However, by inspecting the two for one particular realization in the ensemble, as is done in Fig. 3b and d, we observe that the Haar fluctuation can present a break in the curve that visually is much more pronounced than in the corresponding Haar wavelet variance. This is nothing but a visual illusion (the two plots contain the same information); a curve that changes its slope from negative to positive is more easily perceived to represent different qualities than the curve that only changes its positive slope somewhat. Since all the curves are produced from realizations of the fGn, all scale breaks are spurious, and caused by the diverging statistical uncertainty in the high-scale end. Thus, if the underlying scaling is close to $1 / f$ noise, then weak trends or oscillations tend to appear as breaks in the Haar fluctuation curve but are much less visible in the wavelet variance curve. A sound approach to graphical tools like this should avoid visualizations like the Haar fluctuation, which exaggerates such spurious breaks. 

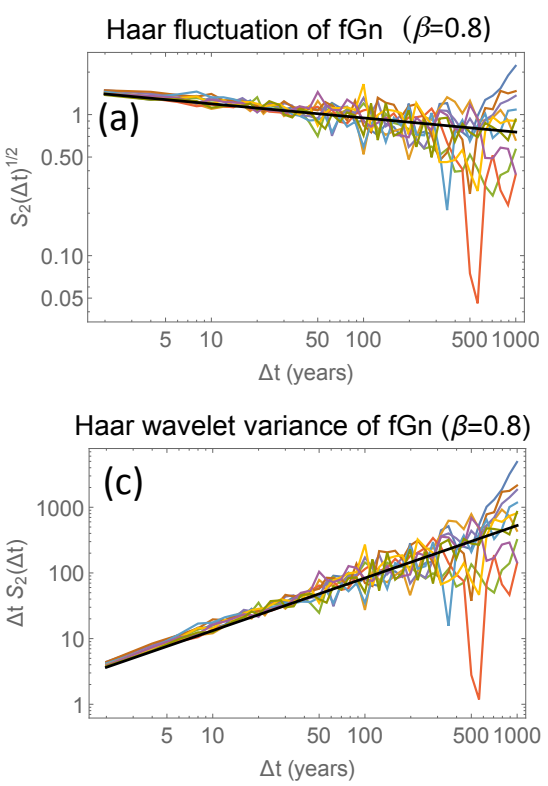
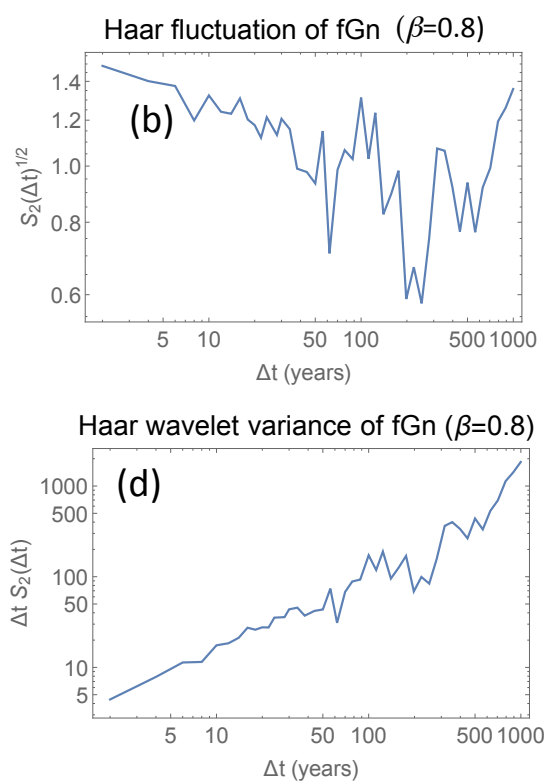

Figure 3. (a) The Haar fluctuation of 20 realizations of an fGn with $\beta=0.8$. (b) The Haar fluctuation of one realization in the ensemble. (c) The Haar wavelet variance of the same 20 realizations as in (a). (d) The Haar wavelet variance of the same realization as in (b).
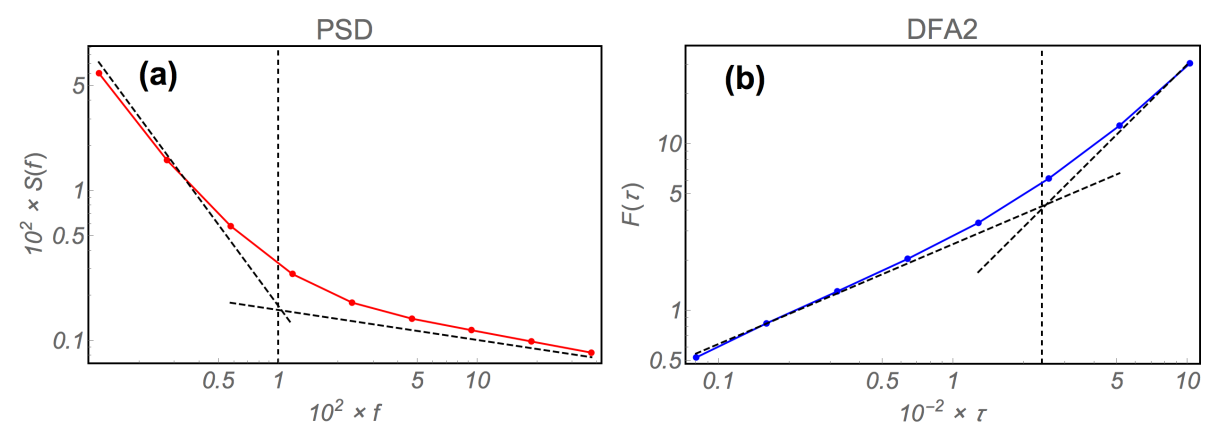

Figure 4. (a) The power spectral density of an ensemble mean of synthetic processes comprised of a superposition of a white noise $(\beta=0.2)$ and a Brownian motion $(\beta=1.8)$. (b) DFA fluctuation function $F_{2}(\tau)$ for ensemble mean of the same process. The dashed lines are the limiting slopes at short/long scales. Their intersection is used to define a transition frequency (vertical dashed line) between the two scaling regimes in (a) and a transition scale for the DFA in (b).

\subsection{The detrended fluctuation analysis (DFA)}

This estimator was invented by Peng et al. (1994) and first applied to climatic time series by Koscielny-Bunde et al. (1996). The technique performs an $n$ th-order polynomial detrending of the cumulative sum of the data record on each timescale $\tau$, and computes the variance (fluctuation function) $F_{n}(\tau)$ of the resulting residual as a function of $\tau$. A good introduction to the technique is Kantelhardt et al. (2001).

DFA performs very well when it comes to estimation of the scaling exponent of perfectly scaling signals. Errors arising from the finite length of the data record are smaller than for other estimators. However, since the nature of DFA is to perform detrending on all scales, the variance on a scale $\tau$ is strongly underestimated compared to the actual variance, even when the signal is perfectly scaling with no imposed trend. The method works for estimation of the scaling exponent because (in a perfectly scaling signal) this underestimation is the same on all scales. This underestimation of the true variance increases with degree of detrending (the order $n$ of the polynomial), so even if the slopes of the curves $\log F_{n}(\tau)$ vs. $\log \tau$ remain the same as $n$ increases, the variance goes down, as shown for instance in Fig. 4 of Kantelhardt et al. (2001). In effect $F_{n}(\tau)$ does not measure the variance on scale $\tau$, but is rather a weighted sum of variances on shorter scales than $\tau$.

For this reason DFA shifts a scale break to longer scales, as is shown by Fig. 4c in the paper by Kantelhardt et al. (2001). We illustrate this in Fig. 4, where we have employed DFA to an ensemble of realizations of length 2000 time steps of a process which is a superposition of an fGn with $\beta=0.2$ and an $\mathrm{fBm}$ with $\beta=1.8$. The power spectrum exhibits a 
Table 1. Results using approach 1 for multiproxy temperature reconstructions.

\begin{tabular}{lrcccl}
\hline Reconstruction & Time period & $\beta_{1, \text { data }}$ & $\begin{array}{c}\text { Conf. range } \\
\text { for } \beta_{1, \mathrm{MC}}\end{array}$ & $\beta_{2, \text { data }}$ & $\begin{array}{c}\text { Conf. range } \\
\text { for } \beta_{2, \mathrm{MC}}\end{array}$ \\
\hline Jones et al. (1998) & $1000-1850$ & 0.5 & $(0.4,0.7)$ & 1.2 & $(-0.8,1.7)$ \\
Briffa et al. (2001) & $1402-1850$ & 0.6 & $(0.4,0.8)$ & 2.9 & $(-2.0,3.0)$ \\
Esper et al. (2002) & $831-1850$ & 1.3 & $(0.8,1.2)$ & 1.2 & $(0.2,3.3)$ \\
Huang (2004) & $1500-1850$ & 0.7 & $(0.6,1.0)$ & 2.3 & $(-4.4,6.0)$ \\
Moberg et al. (2005) & $0-1850$ & 0.8 & $(0.6,1.0)$ & 1.2 & $(0.0,1.5)$ \\
Mann et al. (2008) & $500-1850$ & 2.5 & $(1.9,2.6)$ & 1.6 & $(1.5,3.1)$ \\
Neukom et al. (2014) & $1000-1850$ & 0.6 & $(0.4,0.8)$ & 1.3 & $(-0.8,1.9)$ \\
\hline
\end{tabular}

clear transition in the scaling with the centre of the break located at the frequency $f \approx \times 10^{-2}$, corresponding to scale $\tau \approx 1 / f=100$. This break is shifted towards a larger scale $(\tau \approx 250)$ in DFA $(n=2)$, which is the DFA order employed in Rybski et al. (2006). This means that a scale break at $\tau \approx 100$ years would appear in DFA of a 2000-year record as a break at $\tau \approx 250$ years. But in Rybski et al. (2006) this is roughly the maximum timescale investigated by DFA for the various multiproxy records; hence, such a break could not have been detected by the methods employed in that paper. This demonstrates that DFA does not have the sensitivity on long timescales to detect scale breaks unless the break takes place at a transition scale which is at least an order of magnitude shorter than the length of the observed data record. For this reason DFA is not employed as an estimator in the present paper.

\section{Data}

The scaling is analysed in seven proxy/multiproxy temperature reconstructions representing late Holocene temperature and one temperature reconstruction representing the entire Holocene time period, in addition to six reconstructions of stable isotope ratios from the deep ice cores GRIP, GISP2, and NGRIP from Greenland, and EPICA, Taylor dome, and Vostok from Antarctica. Information and analysis results from GISP2, NGRIP, Taylor, and Vostok are provided in Sect. S2. From the available ice-core time series we extract sub-series covering only the Holocene and only the last glacial period, respectively. For the GRIP ice core we also extract a time series covering $0-85 \mathrm{kyr}$ BP. Since the exact timing of the transition between the Holocene and the last glacial period is slightly different for Greenland and Antarctica, we have chosen the start and end of the time series carefully for each series, such that the transition is not contained in any of the "Holocene-only" or the "glacial-only" time series.

\subsection{Proxy/multiproxy late Holocene temperature reconstructions}

We have chosen seven proxy- or multiproxy-based temperature reconstructions for our study, and in order to avoid the trend effect from anthropogenic warming we have discarded data after AD 1850 (see Table 1). All time series are given with annual resolution. A few of the reconstructions are based partly on the same raw proxy records, but we include all since the reconstruction methods are different. The Jones et al. (1998) multiproxy reconstruction represents Northern Hemisphere temperature. The Briffa et al. (2001) reconstruction represents the continental region $20-90^{\circ} \mathrm{N}$ and is constructed from tree rings. The Esper et al. (2002) reconstruction is also based on tree rings and represent the continental region $30-80^{\circ} \mathrm{N}$. The Huang (2004) reconstruction is based on borehole temperatures, integrated with instrumental temperatures and the multiproxy reconstruction by Mann et al. (1999). The Moberg et al. (2005) multiproxy reconstruction represent Northern Hemisphere temperature, and is smoothed on the shortest timescales, so estimates of the scaling exponents are restricted to timescales from 4 years and longer. The Mann et al. (2008) multiproxy reconstruction represents global temperature, and is smoothed up to decadal timescales. The Neukom et al. (2014) multiproxy reconstruction represents Southern Hemisphere temperature.

\subsection{Multiproxy full Holocene temperature reconstruction}

The temperature reconstruction described in Marcott et al. (2013) covers the entire Holocene time period. The reconstruction is based on 73 proxy records with temporal resolution varying from 20 to 500 years. The spatial distribution of proxy data is near global, and there is a high percentage of data sets from marine sites. The proxy records had been interpolated to 20 -year resolution before constructing the temperature reconstruction, and the final record is presented with a 20 -year resolution. The reconstructed temperature gets gradually smoother as one goes back in time. This is observed from the time series itself in Fig. 9a. From the Supplement of the Marcott et al. (2013) paper it is clear that the proxy records covering the most recent time also in general exhibit the best temporal resolution. The reconstructed temperature data for the past 1500 years therefore represent high-frequency variability in a more realistic way than the remaining part of the reconstruction. 

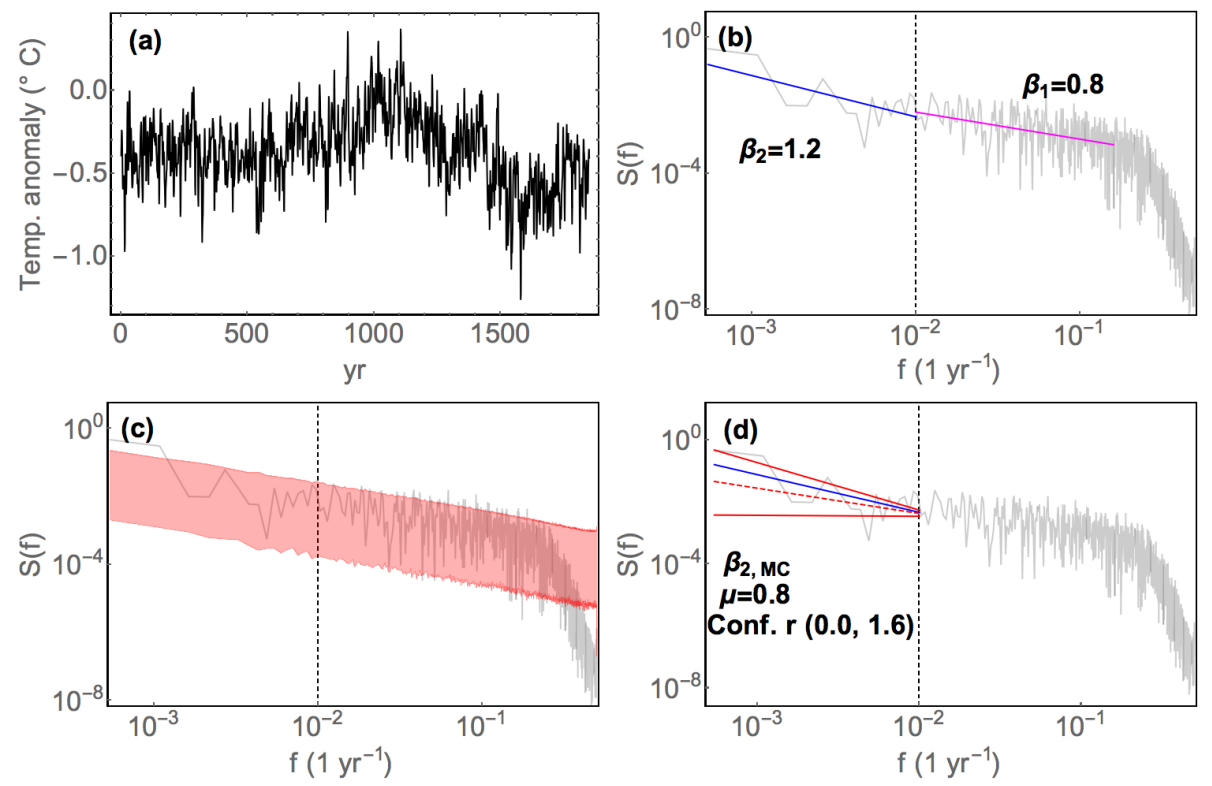

Figure 5. (a) The Moberg et al. (2005) reconstructed temperature for the Northern Hemisphere. (b) Estimated values of $\beta_{1}$ and $\beta_{2}$. (c) $95 \%$ confidence range for periodograms in the Monte Carlo study. (d) $95 \%$ confidence range for estimates of $\beta_{2}$.

\subsection{The GRIP ice core}

The European multinational research project "Greenland Ice Core Project" (GRIP) completed drilling a $3028 \mathrm{~m}$ deep ice core from central Greenland in 1992 (Dansgaard et al., 1993). Two GRIP data sets are used in this study, one with high temporal resolution covering 0-91 kyr BP (Ditlevsen et al., 1996), and one with lower temporal resolution covering 0250 kyr BP (Greenland Ice Core Project, GRIP; Johnsen et al., 1997). The high-resolution data set was provided by Peter Ditlevsen at the Centre for Ice and Climate, Niels Bohr Institute, University of Copenhagen (personal communication, 2015). Both data sets are used to estimate the spectral exponents, but the results shown in Sect. 5 are for the highresolution time series. Both temperature reconstructions are based on $\delta^{18} \mathrm{O}$.

\subsection{The EPICA Dome C ice core}

The European Project for Ice Coring in Antarctica (EPICA) drilled two deep ice cores in Antarctica between 1996 and 2006. Here we focus on the core from dome $\mathrm{C}$ at the East Antarctic Plateau (abbreviated EDC hereafter), covering the past 740000 years (EPICA community members, 2004; Jouzel et al., 2007). The temperature reconstruction is based on $\delta \mathrm{D}$.

\section{Results}

\subsection{Results for late Holocene multiproxy reconstructions}

Three approaches are used to detect a scale break from the spectra of the seven multiproxy temperature reconstructions. The first is to assume a scale break at exactly 100 years, and then estimate $\beta$ for long and short timescales and determine the uncertainties for each estimate. By this approach we demonstrate that scale breaks may occur by chance from a monoscaling model, without being statistically significant. The second approach is to use a procedure for automatic detection of a scale break from a two-scaling regimes hypothesis, and show that a wide range of timescales $\tau_{c}$ for the break, and a wide range of scaling exponents $\beta_{1}$ and $\beta_{2}$ arise by applying the procedure to a Monte Carlo ensemble of monoscaling time series. We also employ the structure function approach, and show that the enhanced power at large scales is associated with an oscillation with characteristic scale around 500 years.

Figure 5 illustrates the procedure and results for the Moberg temperature reconstruction, using the first approach. The spectral exponent $\beta$ is estimated from the standard periodogram of the reconstructed data, for timescales shorter than $10^{2}$ years $\left(\beta_{1, \text { data }}\right)$ and for timescales longer than $10^{2}$ years $\left(\beta_{2, \text { data }}\right)$, as shown in Fig. 5b. A Monte Carlo (MC) ensemble of synthetic fGn's with 2000 members is then con-

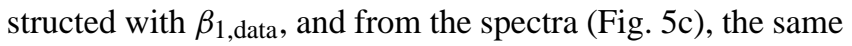
estimation technique is used to estimate $\beta_{1, \mathrm{MC}}$ and $\beta_{2, \mathrm{MC}}$ for each realization. From the distribution of the estimated $\beta_{1, \mathrm{MC}}$ and $\beta_{2, \mathrm{MC}}$, the $95 \%$ confidence ranges are computed. Figure $5 \mathrm{~d}$ shows the mean and $95 \%$ confidence range for $\beta_{2, \mathrm{MC}}$. 
Table 2. Results using approach 2 for multiproxy temperature reconstructions.

\begin{tabular}{lccrr}
\hline Data set & $\beta_{1, \text { data }}$ & $\beta_{2, \text { data }}$ & $\begin{array}{r}\tau_{c} \\
\text { (year) }\end{array}$ & $\beta_{2}-\beta_{1}$ \\
\hline Jones et al. (1998) & 0.5 & 0.9 & 38 & 0.4 \\
Briffa et al. (2001) & 0.9 & 0.2 & 22 & -0.7 \\
Esper et al. (2002) & 1.4 & 1.0 & 38 & -0.4 \\
Huang (2004) & 0.8 & 2.2 & 94 & 1.4 \\
Moberg et al. (2005) & 0.7 & 2.6 & 353 & 1.9 \\
Mann et al. (2008) & 3.1 & 0.9 & 47 & -2.2 \\
Neukom et al. (2014) & 0.5 & 0.8 & 9 & 0.3 \\
\hline
\end{tabular}

Since the blue line $\left(\beta_{2, \text { data }}\right)$ is within the confidence range for a MC ensemble of fGn's with $\beta=0.8$, the single-scaling regime hypothesis cannot be rejected. Results for all seven reconstructions are shown in Table 1.

For the Esper at al. (2002) reconstruction the estimate of $\beta_{1, \text { data }}$ is slightly outside the confidence range, but this is due a bias of the synthetic $\mathrm{fBm}$ for $\beta$ slightly higher than unity (see Sect. S1.1 for further details). This deviation should therefore be ignored.

From the second approach we obtain for each reconstruction two values of $\beta$ and a time for the scale break. The procedure is to fit two line segments with slopes $\beta_{1}$ and $\beta_{2}$ to the $\log -\log$ spectrum, such that they join at $f=f_{c}=1 / \tau_{c}$. The two slopes and the transition frequency $f_{c}$ are the parameters to be fitted by an ordinary least-squares procedure. Results for the seven temperature reconstructions are provided in Table 2, where the differences in $\beta$ values are also included. The scale-break hypothesis of Lovejoy and Schertzer (2012b) states that the difference $\beta_{2}-\beta_{1}$ should be around unity. This procedure has also been tested on a Monte Carlo ensemble of monoscaling fGn's. Figure 6 shows a histogram of the differences in estimated $\beta_{2}$ and $\beta_{1}$. The histogram shows that the scale breaks detected by this procedure in the multiproxy records are not unlikely to be detected in records with a single scaling regime, i.e. their detection does not reject the single-scaling regime hypothesis. A histogram of $\tau_{c}$ also shows a broad distribution (figure not shown).

In Fig. 7a we plot SFs for the cumulative sum of the Moberg multiproxy reconstruction. The SFs are straight in the $\log -\log$ plot up to around 500 years, but then there is a broad bump. By examining the record it becomes apparent that this bump is associated with an oscillation with a period of the order of a millennium that involves the Medieval Warm Anomaly (MWA) high and the Little Ice Age (LIA) low. The fact that this oscillation shows up in the high-order SFs indicates that its amplitude is larger than consistent with the underlying persistent noise. If we fit the SFs by straight lines up to 500 years we obtain the scaling function in Fig. 7b with slope $H \approx 0.87$, in very good agreement with what was found from the instrumental data. If this oscillation were a manifestation of a new scaling regime with $\beta>1.4(h>0.2$,

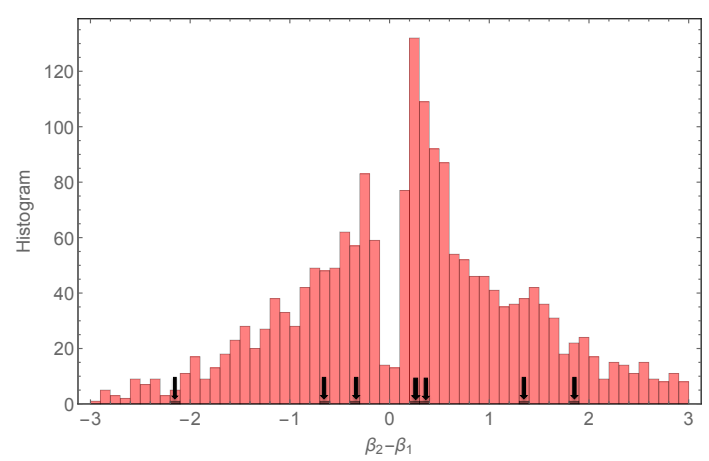

Figure 6. Differences in $\beta_{2}$ and $\beta_{1}$ for a Monte Carlo ensemble with 2000 members of synthetic LRM processes with $\beta=0.7$. The black arrows indicate the differences from the multiproxy reconstructions.

$H>1.2$ ), we should expect the SFs in Fig. 7a to be straight with slope $q$ on scales $>\tau_{c}$, and the scaling function obtained by fitting lines to the SFs on these scales to have slope $H=1$ (this is easily demonstrated by Monte Carlo simulations). What we observe, however, is a downward bend caused by the oscillation discussed above. It can be correctly argued that this bend is not statistically significant, since we only have one sample of it, but it demonstrates very clearly that there are cases where fluctuation measures like the periodogram or the Haar fluctuation function will suggest a new scaling regime with higher scaling exponents (see Fig. 8b), while the SF method will suggest oscillations.

The issue of modelling the fluctuations on a multi-century timescale as a second scaling regime or an oscillation is illustrated in Fig. 8. The idea is the same as in Fig. 2, but now the "trend" is modelled as a growing oscillation on the form At $\sin [\omega(t-\varphi)]$. The Moberg record and the fitted trend shown in Fig. 8a and b show the Haar fluctuation of the record along with realizations of a model comprised of a linear combination of an fGn and an fBm (red curves), and realizations of a model comprised of a linear combination of an fGn and the oscillatory trend. Again, the observation is consistent with both models, but the latter exhibits smaller uncertainties at longer scales and hence is a better statistical model. The attribution study of Rypdal (2015) points at a combination of volcanic and solar forcing, with greater weight to volcanoes, as the main driver of this oscillation. Rypdal and Rypdal (2014) show that the residual after subtracting the response to this forcing is well modelled as an fGn with $h$ compatible with what was used for the short scales in Fig. 8b. Thus, compared to a two-scaling regime model, the simpler and more accurate statistical model of the Moberg record is to model the internal variability as a persistent fGn for all scales up to the length of the record, superposed on a forced oscillatory trend.

An important feature shown by the scaling functions in Figs. 1 and 7 is that the background noise in GMST is monofractal. It is also Gaussian. This means that a frac- 

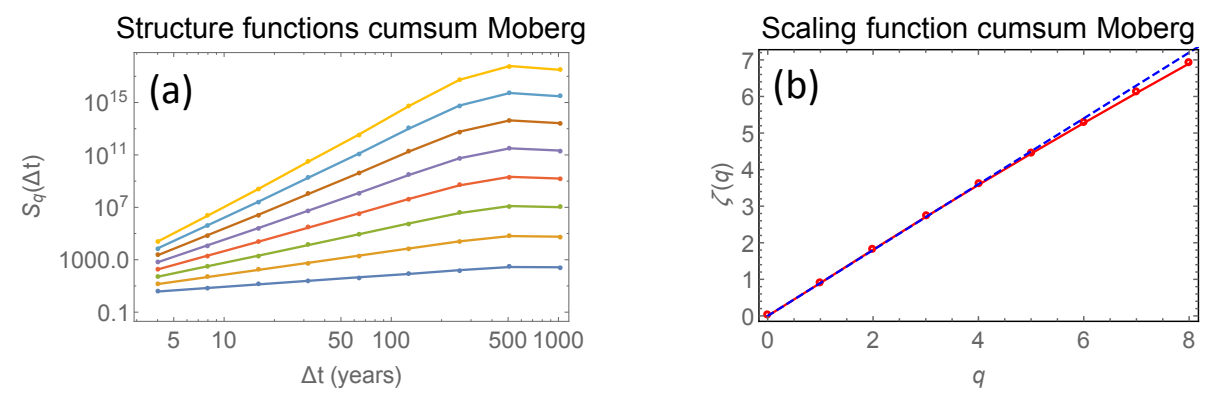

Figure 7. (a) Structure functions for the cumulative sum of the Moberg NH reconstruction year 0-1979. (b) Scaling functions for the cumulative sum computed from straight line fits to the SFs in the scale range 1-500 years.
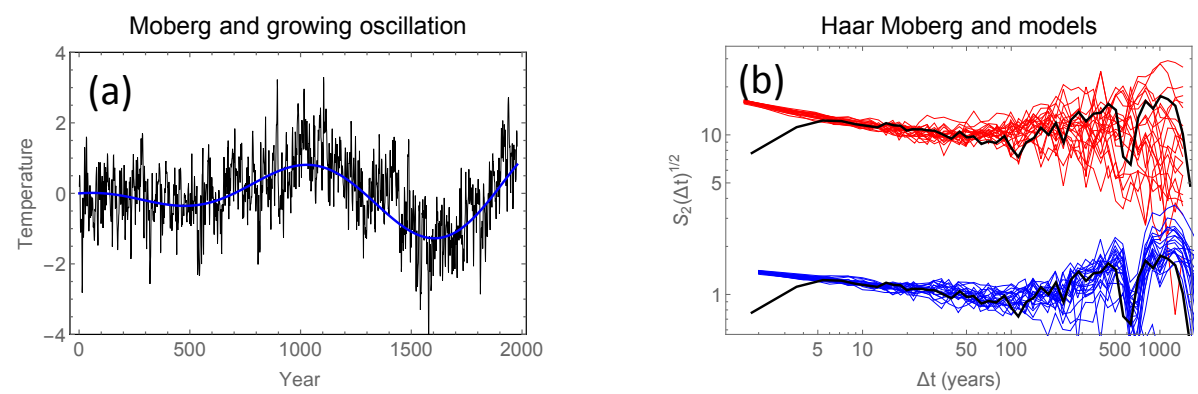

Figure 8. (a) The Moberg reconstruction (black). A fit of the function $A t \sin [\omega(t-\varphi)]$ (blue). (b) Black curves are the Haar fluctuation function of the Moberg record; the upper curve is multiplied by 10. The red curves are Haar fluctuation functions of 20 realizations of a model comprised of a linear combination of an fGn with $\beta=0.6$ and an $\mathrm{fBm}$ with $\beta=1.6$. The blue curves are the same of a model comprised of a linear combination of an fGn with $\beta=0.6$ and the trend $A t \sin [\omega(t-\varphi)]$.

tional Gaussian noise is a good model for these fluctuations, and hence that all essential information is contained in the scaling exponent and the variance. This is also true for ice-core data in the Holocene, while during the last glaciation, ice-core data are neither monofractal nor multifractal. In an accompanying paper from our research group (Rypdal and Rypdal, 2016) it is demonstrated that if the transitions between stadial and interstadials associated with DO events and glacial-interglacial transitions are removed from the ice-core records, the remaining fluctuations scale roughly as a $1 / f$ noise $(\beta \approx 1)$ on a timescale longer than a century. In other words, over the length of the Antarctic ice-core record ( $800 \mathrm{kyr})$ the temperature variability can be described as a series of glacial-interglacial transitions and, within the glacial periods, a series of stadial-interstadial transitions, superposed on a background $1 / f$ noise. This suggests that Holocene variability should also exhibit this scaling of the climate noise, and the analysis of the Moberg record we have made here does not reject that hypothesis.

\subsection{Results for the full Holocene multiproxy reconstruction}

The reconstruction by Marcott et al. (2013) has been analysed with the periodogram in a particular way to take into account the increasing smoothness of the record as one goes backward in time. The wavelet scalogram in Fig. 9b illustrates that the wavelet power on long timescales is substantially higher in the early Holocene as compared to the later half. If we compute the standard periodogram for the full time series, the resulting spectral exponent is $\beta=2.9$. The power is artificially low at high frequencies, and this is corrected by dividing the time series into segments $S_{n}$ of lengths $2^{n} \times 400$ years, with $n=0,1,2, \ldots, 5$, and starting with the most recent period. Hence, $S_{1}=50$ $450 \mathrm{yr} \mathrm{BP}, S_{2}=50-850 \mathrm{yr} \mathrm{BP}, S_{3}=50-1650 \mathrm{yr} \mathrm{BP}, S_{4}=50$ $3250 \mathrm{yr} \mathrm{BP}, S_{5}=50-6450 \mathrm{yr} \mathrm{BP}$, and $S_{6}=50-11290 \mathrm{yr} \mathrm{BP}$ (longest possible record, shorter than $2^{5} \times 400$ ). The periodogram was estimated for each segment, and then a new power spectrum was created using only parts of each segment assumed to be trustworthy with regard to preserved variability. All of $S_{1}$ was included, while for $S_{2}, \ldots, S_{6}$ only the low-frequency parts were included (none overlapping). By this composition, the resulting power spectrum represents the variability on all timescales more correctly. Figure 9c shows the spectra of all six segments, in addition to the corrected spectrum (blue dots, black line). The value of $\beta=1.3$ is estimated from this line. The corrected spectrum still does not represent the true scaling of the global temperature, but it is a better representation than the periodogram of the original record. 


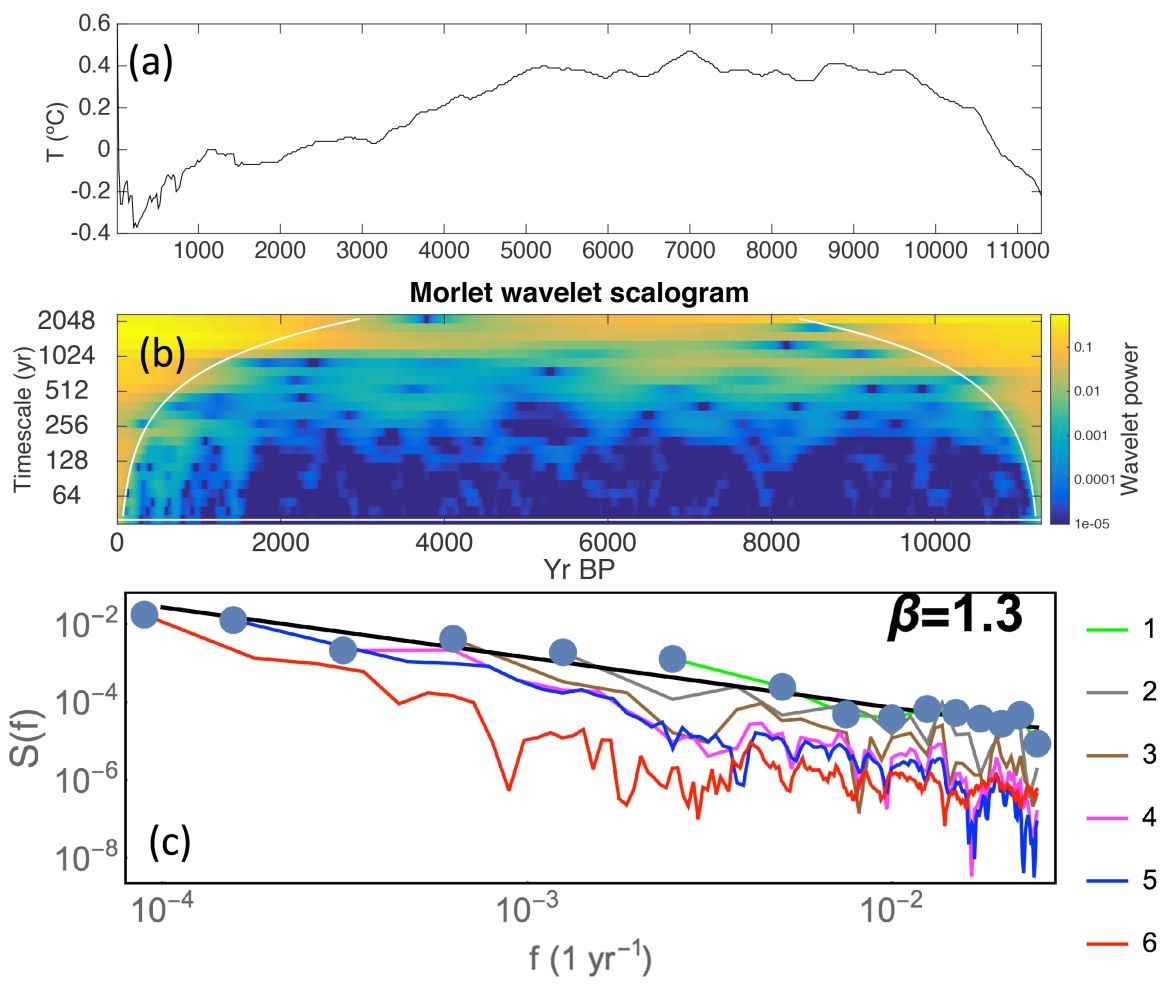

Figure 9. (a) The reconstructed time series covering the entire Holocene time period. (b) Wavelet scalogram for the same time period. (c) Section 1-6 of the reconstruction described in Sect. 5.2, and composite spectrum (black line, blue dots). The estimated $\beta$ is estimated for this line.

\subsection{Results for ice-core time series}

For the time series plots, time on the horizontal axis is given in years BP (before present), where "present" is defined as AD 1950. The spectral analysis is presented in a double-logarithmic plot. The raw periodogram is plotted in grey, while the log-binned version is marked by black points. The spectral index $\beta$ is estimated from the log-binned periodogram in the region shown by the blue line. Finally, the blue, shaded area indicate the $95 \%$ confidence range estimated from an ensemble of synthetic fGn's/fBm's with $\beta$ and variance estimated from the log-binned periodogram. The plot of the wavelet scalogram is included in this section only for the GRIP Holocene/past $85 \mathrm{kyr}$ record.

For the last glacial period, we present time series and periodograms for a time interval of $\approx 80 \mathrm{kyr}$. Spectral analysis results are also included for a combined Holocene-last glacial period time series from the GRIP ice core to illustrate that analysis of such records will be dominated by the glacial climate and suppress the characteristics of Holocene climate.

\subsubsection{Results from the GRIP ice core}

Figure 10a shows the $\delta^{18} \mathrm{O}$ time series of the Holocene part of the high-resolution GRIP ice core, and Fig. 10b the periodogram from the same time series. Figure 10c displays the same time series as shown in Fig. 10a, but with the earliest 2500 years removed. Figure $10 \mathrm{~d}$ shows the periodogram for the time series in Fig. 10c. The rationale for removing the earliest part of the Holocene record can be seen from Fig. 10a, where one observes a decrease in $\delta^{18} \mathrm{O}$ around $8 \mathrm{kyr}$ BP. This particular decrease is often observed in palaeotemperature records from the Northern Hemisphere, and especially in records from the North Atlantic region. The feature is known as the $8.2 \mathrm{kyr}$ event, and the temperature change was probably caused by a large pulse of freshwater into the North Atlantic Ocean associated with the collapse of the Laurentide ice sheet (Alley and Agustsdottir, 2005). In Fig. $10 \mathrm{~b}, \beta$ is estimated to be $\approx 0.3$ for timescales up to $10^{3}$ years. No scale break is detected on centennial timescales. The low value of $\beta$ is typical for local temperature data from continental sites (Blender and Fraedrich, 2003; Fraedrich and Blender, 2003). On timescales longer than a millennium we can infer a higher $\beta$, but still $\beta<1$. Since the $8.2 \mathrm{kyr}$ event might affect the scaling we also analysed the shorter record (Fig. 10c). The periodogram for this time series is essentially flat. Figure 11 shows the Mexican hat and Morlet wavelet scalograms for the full Holocene section of the GRIP ice core. The $8.2 \mathrm{kyr}$ event clearly increases the power at millennial timescales, and this event is the source of the increased power observed at that timescale in Fig. 10b. From the pe- 
riodogram of the Holocene part of the low-resolution GRIP time series we estimate $\beta \approx 0.1$ (not shown in figure).

Lovejoy and Schertzer (2012b) claim that the palaeotemperature records from Greenland ice-core data are exceptionally stable for the Holocene period, and therefore downplay the significance of the low scaling exponent and the absence of the scale break. This point of view is repeated in Lovejoy and Schertzer (2013), where an additional palaeo-seasurface temperature (SST) reconstruction is analysed (Berner et al., 2008). This SST reconstruction is based on diatoms from a marine sediment core sampled at Reykjanes Ridge in the western North Atlantic Ocean. Lovejoy and Schertzer (2013) find the spectral exponent $\beta=1.4$. When we analyse the same data we find one single scaling regime and $\beta=1.1$. There is nothing strange or exceptional in this land-sea difference. The same pattern of high/low spectral exponents are found for ocean/land surface temperature data for the instrumental period; see Fredriksen and Rypdal (2016) for spectral analyses of numerous instrumental, reanalysis, and GCM data sets. Figure 5 in that paper is particularly illustrative, as it shows that Reykjanes Ridge is located in a region with exceptionally high $\beta$.

Continuing the analysis of the GRIP palaeotemperature record, Fig. 12a displays the $\delta^{18} \mathrm{O}$ time series from the last glacial period, and Fig. 12b the periodogram for the same time period. In Fig. 12a the DansgaardOeschger (DO) events are observable as rapid warming over decadal timescales, followed by more gradual cooling (Bond and Lotti, 1995). In Fig. 12b we find $\beta \approx 1.8$ for timescales longer than $10^{2}$ years and shorter than $10^{4}$ years. On centennial timescales and shorter, the spectrum is flatter. This means that a hypothesis of a scale break at centennial time scales is plausible under glacial climate conditions, even though such a scale break could not be identified from the Holocene time series. From the low-resolution GRIP data set we estimate $\beta \approx 1.3$ for timescales longer than centennial, and a scale break is seen at this scale (figure not shown).

Figure 13a shows the past $85 \mathrm{kyr}$ time series of the highresolution GRIP ice core, and Fig. 13b the periodogram for the same time series. In Fig. $13 b, \beta \approx 1.6$ for time scales longer than centennial, and a scale break is visible at this scale. The periodogram in Fig. 13 is very similar to that in Fig. 12, indicating that the information from the Holocene is suppressed to a high degree in the periodogram of this time series.

\subsubsection{Results from the EDC ice core}

Figure 14a shows the Holocene time series of the EDC ice core, and Fig. 14b the periodogram for the same time series. In Fig. 14a the Antarctic equivalent to the Northern Hemisphere Holocene climate optimum (HCO) occurred between 11500 and $9000 \mathrm{yr}$ BP (Masson et al., 2000). In Fig. 14b, $\beta \approx 0$ for timescales shorter than $10^{3}$ years.
Figure 15a shows the time series of the EDC ice core from the last glacial period, and Fig. 15b the periodogram for the same time period. We observe from the time series that the fluctuations do not coincide with the DO events in the GRIP ice core with respect to timing and amplitude. Like the glacial part of the GRIP ice core the EDC glacial time series Fig. 15a has higher fluctuation levels than the Holocene counterpart. In Fig. $15 \mathrm{~b}$ we estimate $\beta \approx 1.6$ for $10^{3}$ years $<\tau<10^{4}$ years. The scale break in these figures appears at $10^{3}$ years.

\section{Discussion and conclusions}

In this paper we have examined a number of palaeoclimatic temperature records to assess the feasibility of detection with confidence of multiple scaling regimes in Holocene climate, and in particular a break in scaling around centennial timescales. Seven proxy/multiproxy reconstructions from the late Holocene, and one for the entire Holocene, have been selected for analysis due to high temporal resolution and coverage in time, and six reconstructions from deep ice cores sampled at Greenland and Antarctica also meet our requirements for temporal coverage and resolution.

For the seven late Holocene proxy-based temperature reconstructions, our first approach was to assume a break at exactly 100 years. Obviously there are few data points available for estimation on the longer scales using this procedure, and the estimated values of $\beta_{2}$ are within the uncertainties of a monoscaling model for all seven reconstructions. The scale break is therefore not statistically significant. For the second approach, our systematic procedure detects a break in scaling for all reconstructions. The timescale for the break varies significantly between reconstructions and is in most cases not even located near centennial timescales. The differences $\beta_{2}-\beta_{1}$ vary over a great range and takes on both positive and negative values. This procedure has also been tested on a Monte Carlo ensemble of fGn's and demonstrates that we will find such apparent breaks even in data that should not have breaks.

The discussion and correction of the temperature reconstruction by Marcott et al. (2013) illustrates the potential pitfall of uncritically selecting palaeoclimatic time series for scaling analysis. The time series may be an excellent temperature reconstruction for many purposes, but for scaling analysis one needs to correct for the fact that the data are increasingly low-pass-filtered as one goes backward in time.

From the analyses of the GRIP and EDC ice-core data, a scale break at centennial timescales is only observed in records from the last glacial period. Similar results are found for the analysis of four additional ice-core records presented in Sect. S2. The time series for the Holocene from the GRIP and EDC ice cores both exhibit weak persistent scaling (Figs. 10 and 14). The scaling exponent is estimated to $\beta \approx 0.3$ and $\beta \approx 0$ for the two ice cores, respectively, up to 

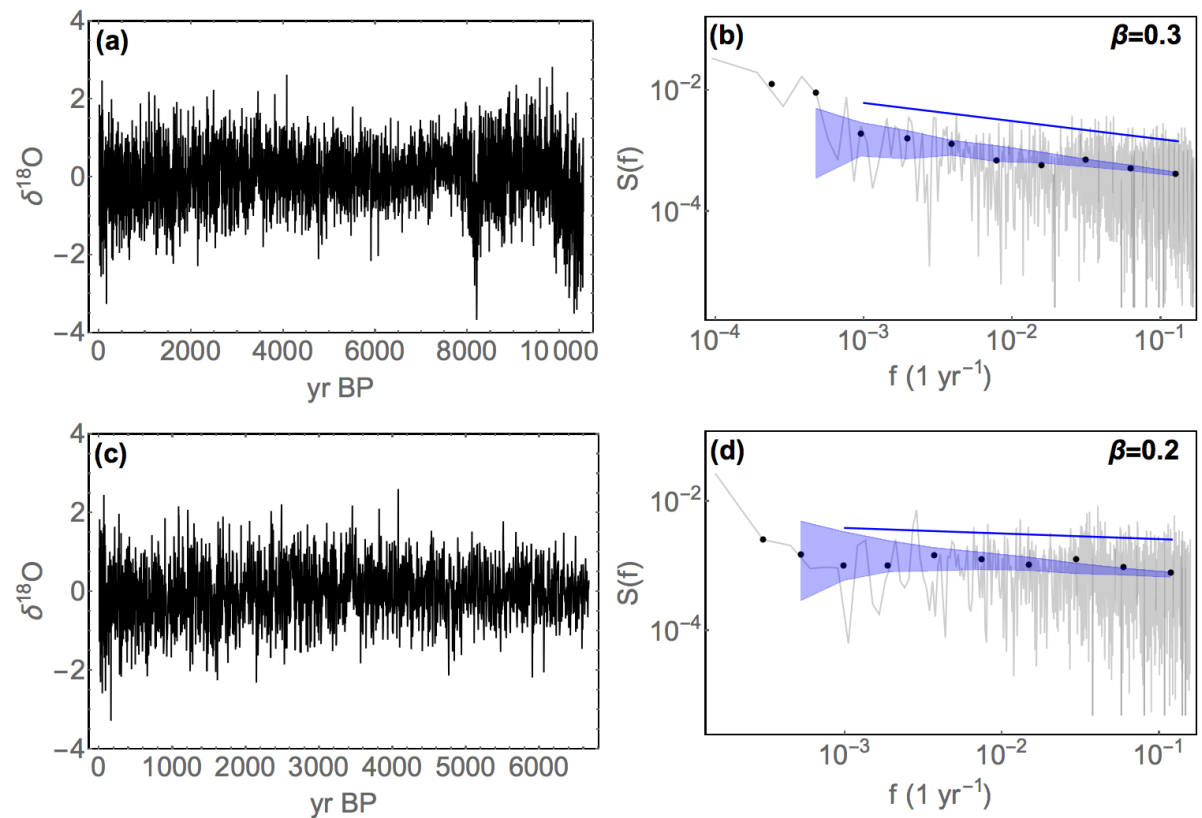

Figure 10. (a) $\delta^{18} \mathrm{O}$ anomalies from the Holocene part of the high-resolution GRIP ice core. (b) Periodogram. The raw periodogram is shown in grey, the log-binned version by black dots. $\beta$ is estimated from the log-binned periodogram in the region marked by the blue line. The confidence range is shown by the blue, shaded area, estimated from a Monte Carlo ensemble of synthetic fGn's with the estimated value of $\beta$ and variance from the log-binned periodogram. (c) Same figure as in (a) except the oldest section has been removed. (d) Periodogram for the time series in (c).
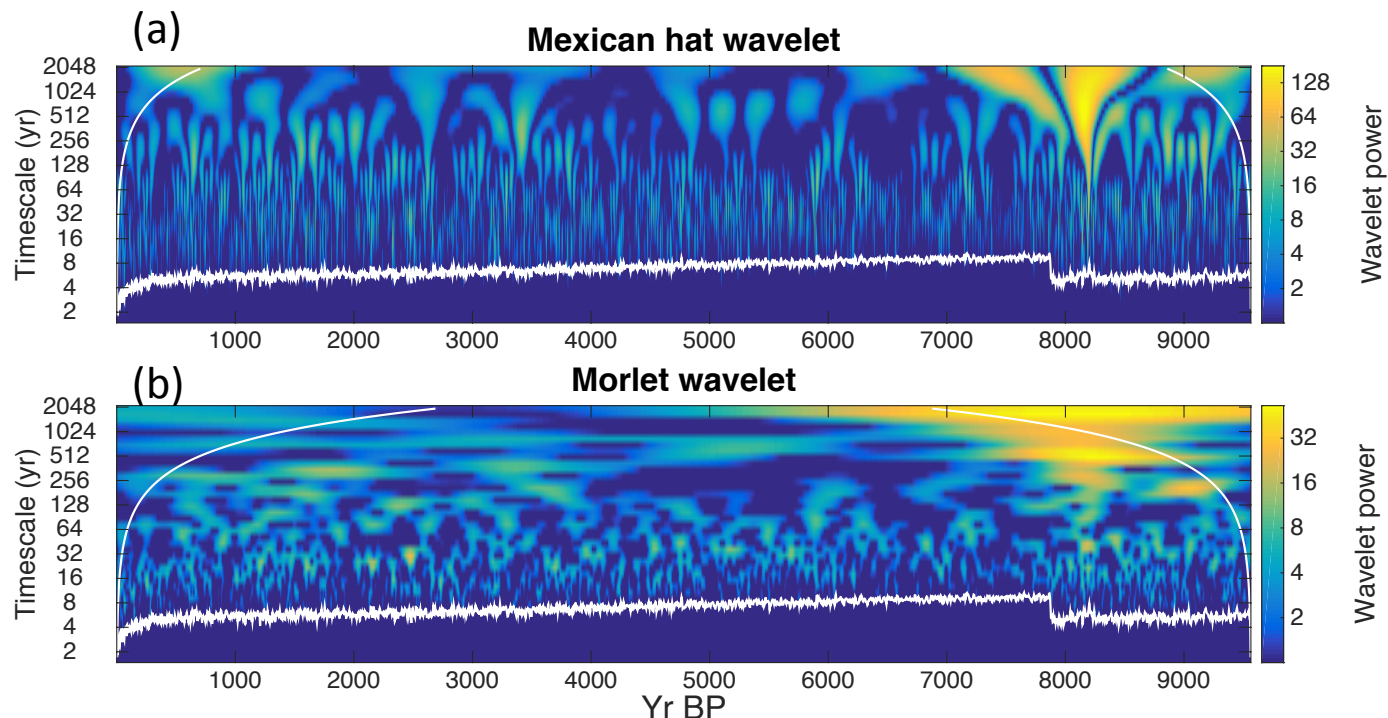

Figure 11. Top panel: the Mexican hat wavelet scalogram for the Holocene part of the GRIP ice core. Bottom panel: the Morlet wavelet scalogram for the same time series. The lower white curve in each plot denotes the varying Nyquist frequency, and the upper white curve the area affected by edge effects. Studies are restricted to the area between the two curves. The colour bar to the right of the figure is used to indicate the magnitude of the wavelet power.

the millennium timescale. No break in scaling can be observed at centennial timescale. The low value of $\beta$ obtained is consistent with the scaling exponents observed over land from the palaeoclimate model run presented in Blender et al.
(2006). On timescales longer than millennial we do not have enough data points to make confident estimates of $\beta$. From the wavelet scalogram we argue that the increase in power 

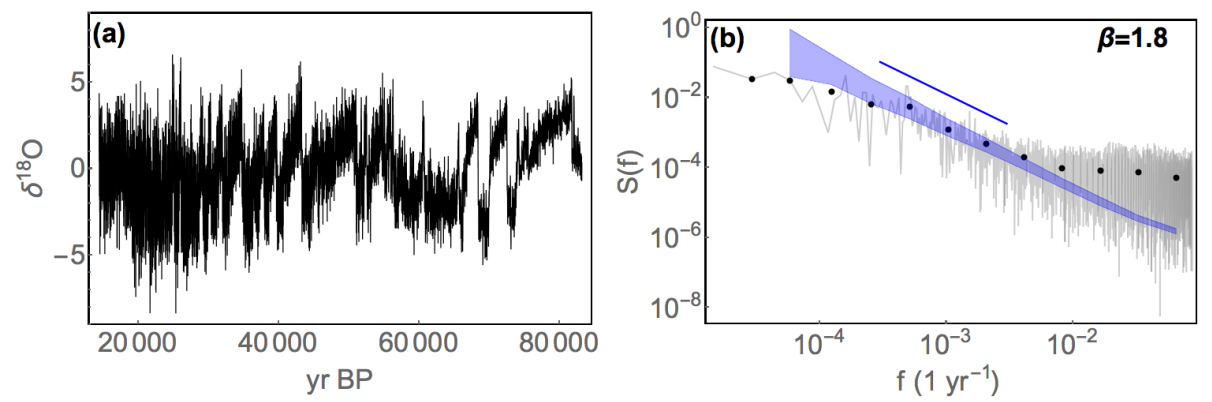

Figure 12. (a) $\delta^{18} \mathrm{O}$ anomaly time series from the last glacial period of the high-resolution GRIP ice core. (b) Periodogram for the time series in (a).
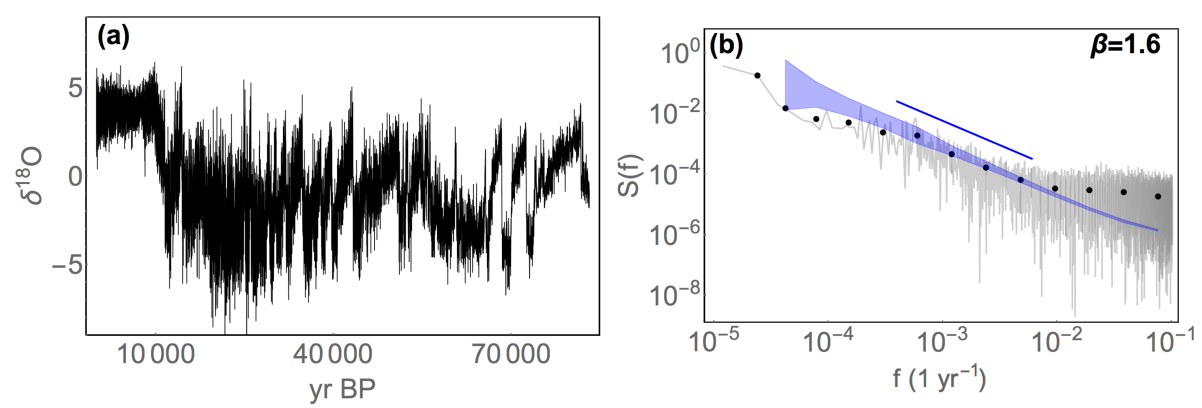

Figure 13. (a) $\delta^{18} \mathrm{O}$ anomalies from the past $85 \mathrm{kyr}$ of the high-resolution GRIP ice core. (b) Periodogram for the same time series.
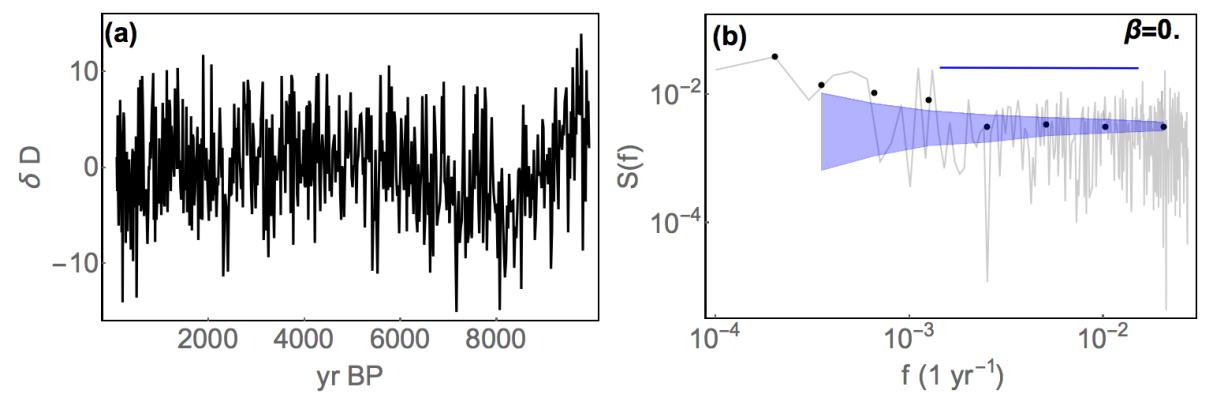

Figure 14. (a) $\delta \mathrm{D}$ anomalies from the Holocene part of the EDC ice core. (b) Periodogram.

seen at the longest timescales in the GRIP periodogram can be attributed to the $8.2 \mathrm{kyr}$ event.

The scaling properties of the GRIP and EDC last glacial period are significantly different from the Holocene. A scale break at centennial timescales is identified with confidence from Figs. 12 and 15. We interpret this scale break as being associated with the variability in Dansgaard-Oeschger events and teleconnections to the Southern Hemisphere (WAIS Divide Project Members, 2015). A number of theories and models exist for the mechanism of these events; see, for example, Dokken et al. (2013). That study indicates that the DO variability is internal and not a direct response to external forcing.

From the GRIP time series including both the Holocene period and the last glacial period we obtain a power spectrum very similar to that of the glacial climate (Fig. 13). Be- cause the durations of the late Quaternary interglacials are generally much shorter than for the glacials, the Holocene temperature variability is strongly suppressed when time series covering $100 \mathrm{kyr}$ or longer are used to estimate scaling exponents. Our analyses of Holocene records, on the other hand, show that a scale break on centennial timescales is not a universal feature, and in those cases in which it appears to be present, it cannot be detected with sufficient certainty.

The DO events are observed exclusively in records from the glacial climate state, but a number of studies investigate the possibility of cyclic climate variability on millennial time scales also in the Holocene. Bond et al. (1997) present palaeoclimatic data from different sources, indicating a number of cooling events throughout the Holocene related to icerafting in the North Atlantic Ocean. The Little Ice Age, the $8.2 \mathrm{kyr}$ event, and even the Younger Dryas are examples of 

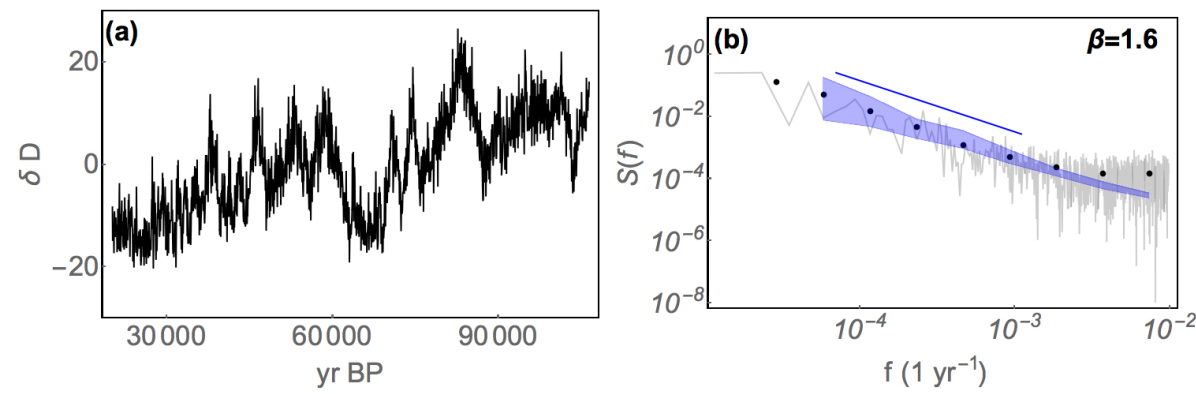

Figure 15. (a) $\delta \mathrm{D}$ anomalies from the last glacial period part of the EDC ice core. (b) Periodogram for the time series in (a).

cold periods sometimes denoted "Bond events". The amplitudes of the temperature anomalies are much smaller than for the DO events, but Bond et al. (1997) find the periodicity to be similar ( $1470 \pm 352$ years, the "Bond cycle"). From these results, the authors infer a pervasive quasiperiodic climate cycle occurring independently of the climate state. The majority of the data indicating Holocene cooling events are from marine sites, and the proxies are in most cases not that sensitive to temperature. Therefore, in the data we have analysed, the evidence for cooling events is essentially limited to the 8.2 kyr event (GRIP) and the LIA (multiproxy reconstructions). If we assume that the DO events cause the characteristic scale break observed in the power spectra at centennial timescale during glacial time periods, should we also expect the Bond events to manifest themselves in the spectra from the Holocene? It is clear from our results that one event in each record is not sufficient to cause a scale break at centennial timescales, but higher power on millennial timescales is possible. The uncertainty due to finite-size effects does not let us draw firm conclusions about the scaling on these timescales.

The idea of a millennial climate cycle, which may have some manifestation even in the Holocene, relates well with the recent results by Rypdal and Rypdal (2016). In this study, the background temperature variability (the climate noise) during the late Quaternary is found to follow a monoscaling power law of $1 / f$ type when DO events and glacialinterglacial transitions are excluded from the analysis. The glacial-interglacial changes are driven by orbital forcing, while the origin of the 1470-year climate cycle is debated and unclear at present. Removing temperature variability related to the Bond cycles for the Holocene may prove an effective way to obtain the proposed $1 / f$ background climate noise. However, it should be mentioned that the very existence of the Bond cycles is debated. Chronological uncertainties in the proxy data, spatial conformity, and undersampling of proxy records are unsettled issues.

Faced with the results we have presented here, one may ask what the practical implications are. Is scaling in climatic time series a useful concept? Our perception is that a scaling law may be useful as a statistical (stochastic) model when a causal description turns out to be very complex, i.e. when the viable alternative is something like a general circulation model. Such a statistical model does not have to exhibit longmemory scaling (a more standard model is a short-memory autoregressive process). Nevertheless, there is strong evidence, for internal variability in surface temperature data, that an fGn is a much better model than an AR(1) process for timescales at least up to centuries (Rypdal and Rypdal, 2014). Thus, for prediction on timescales up to decades, a single-regime monoscaling model with $\beta<1$ is what should be used (Lovejoy et al., 2015). More interesting, however, is whether long-memory scaling is important for prediction on century timescales and beyond, and here the issue of nonstationary scaling $(\beta>1)$ for such timescales becomes crucial. What is the proper value of $\beta$ to use in such prediction efforts in a warming Holocene climate? The conclusion we draw from our results is that, unless we ignore the knowledge that the present climate state of the Earth is an interglacial, we should still use $\beta<1$. One argument that can be raised against that conclusion is that, even though we cannot reject the hypothesis of single-regime scaling based on available Holocene data, we cannot exclude that two scaling regimes is true either. Moreover, the latter is supported from records spanning hundreds of thousands of years which encompass both glacial and interglacial climate. This stalemate reflects that we are faced with a model selection problem where the outcome depends on which available knowledge we prefer to emphasize.

The multiple regime model stresses the information we have on scaling in the second-order statistics such as power spectra $(\beta>1)$ on timescales up to hundreds of thousands of years (Quaternary scaling), and infers that this scaling should be a guideline for prediction independent of whether the initial state is glacial or interglacial. It essentially ignores the fact that Quaternary climate is characterized by several intermittencies (Lovejoy and Schertzer, 2012b). One type of intermittency implies that the probability density functions (PDFs) are heavy-tailed on short timescales and approach Gaussian on longer timescales. This typically happens if the signal is bursty, but without long-range correlation between bursts. In this case the high-order structure functions are not straight lines in a log-log plot. Another, and more restricted, class of intermittent processes comprises 


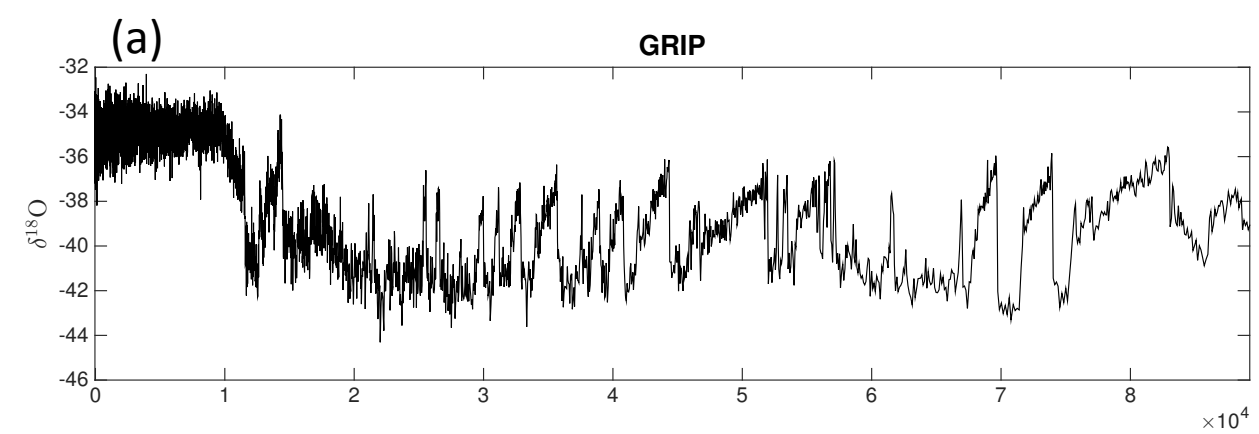

(b)

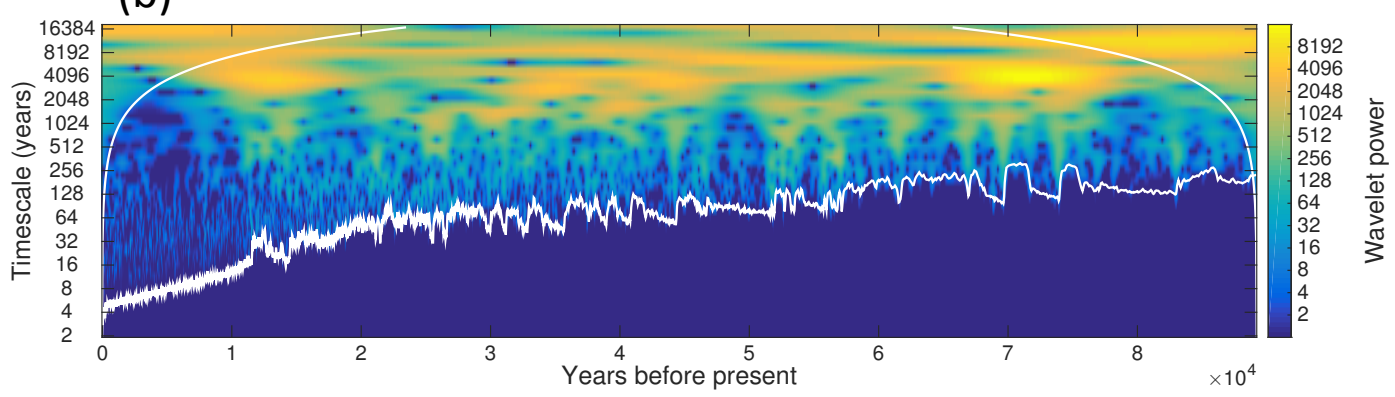

Figure 16. Top panel: the $\delta^{18} \mathrm{O}$ proxy time series for Greenland temperature from the GRIP ice core for the period 0-90 kyr BP. Bottom panel: the Morlet wavelet scalogram for the signal in the upper panel.

those that are multifractal. Here we have correlated bursts and straight structure functions but a curved scaling function. Hence structure functions of higher order than two are needed to characterize the process. For a Gaussian process the power spectral density can be inferred from the secondorder structure function and hence does not convey information beyond second-order statistics. Moreover, Quaternary climate is characterized by the glacial-interglacial transitions, which adds more intermittency, and all this intermittency makes prediction based only on Quaternary scaling very difficult.

The single-regime model, on the other hand, ignores the information available on timescales beyond the Holocene, but makes use of the fact that our present climate state is an interglacial, and that second-order statistics are sufficient to describe the scaling on the timescales that is available to us in the Holocene. As discussed above, and by Rypdal and Rypdal (2016), single-regime scaling can be rejected by data that goes way beyond the Holocene if this scaling is supposed to account for DO events and glacial-interglacial transitions, but there is no statistically significant empirical evidence that the scaling inferred by glacial-state data is present in the interglacial climate state.

The issue discussed in this paper is an example of a more general problem concerning scaling analysis that needs to be addressed in a systematic manner. On geological timescales the Earth is an evolving system. There are cycles, but the Earth rarely repeats itself. The Eemian was similar to the Holocene, but also very different. One striking difference be- ing the evolution of human civilizations and the resulting anthropogenic climate forcing. Thus, the dynamics of the Earth are non-stationary in a very fundamental sense. This makes scaling analysis, and modelling of Earth processes based on such analysis, quite an problematic issue. It has little meaning to talk about a universal scaling in Earths climate since the scaling characteristic on a given range of scales up to a chosen maximal scale $\tau_{\max }$ will depend on the eon, era, period, epoch, or age the analysis is done. In other words, the result will depend on the time $t$ around which the time range $\tau_{\max }$ is centred. A scaling analysis of a given Earthsystem variable must therefore be conditioned by two essential parameters: the range $\tau_{\max }$ of scales considered, and the positioning $t$ of this range in time. The time series and the wavelet scalogram of the GRIP temperature series for the past $t_{\max }=90 \mathrm{kyr}$ illustrates the issue, as shown in Fig. 16 . The central time parameter $t$ is along the horizontal axis and the scale $\tau$ along the vertical. We have no data for the future, which means that the transform cannot be computed correctly above the upper white line in the figure. Likewise, the area below the lower white curve is influenced by the interpolation made due to uneven sampling of the time series. It is apparent that the scalogram is different in the first $11.5 \mathrm{kyr}$ (the Holocene) from the remaining $80 \mathrm{kyr}$ (the last glacial). There is generally lower power on all scales in the Holocene, and the increase in power with increasing scale as $t$ is kept constant is lower. We can also observe from this scalogram that the longest interstadials (warm stages) associated with DO events exhibit variability very similar to the Holocene. 
The literature reveals that there is no consensus of how this issue of non-stationarity could be handled. Ice cores restrict the information we can obtain to somewhat less than $\tau_{\max }=1 \mathrm{Myr}$ BP. This is the range of timescales considered in the work of Lovejoy and Schertzer (2012b), and the period is the Quaternary in which the Earth's climate has been in a bistable state shifting between glacials and interglacials. The methodology and interpretations are based on this choice of the parameters $\left(\tau_{\max }, t\right)$. We do not see anything wrong with that, as long as one is mindful that this is a choice and recognizes that there are other, equally valid, choices. If the issue is understanding of the present and future climate in our present interglacial state, we do not believe this choice is useful, simply because it ignores the knowledge that the Earth at present resides in an interglacial state and probably will continue to do so as long as there is human civilization and anthropogenic forcing on this planet.

The issues dealt with in this paper reveal that a debate on the role of statistical hypothesis testing and scientific method in general is unavoidable if scaling analysis is going to be accepted as a useful method in climate science. Our main statement is that it is scientifically incorrect to draw positive conclusions about an alternative hypothesis (two scaling regimes) from finite size data sets that do not allow rejection of a plausible null hypothesis. This null hypothesis involves a single scaling regime with some distinct events or oscillations superimposed, raising the power on millennial timescale. We do not claim that the scale-break hypothesis is false, but we claim that the null hypothesis cannot be rejected from the data at hand. The distinction between the two hypotheses might seem unimportant, but it is not, because the important issue is the nature of the fluctuations on timescales longer than a few centuries. The scale-break hypothesis states that these fluctuations are scaling. We contend that the data do not reject the null hypothesis that these fluctuations are of quite different nature, and hence that claimed scaling properties on these long timescales cannot be used for understanding or prediction of Holocene climate.

In Lovejoy and Schertzer (2012b) and Lovejoy et al. (2013) the two-regime scaling hypothesis is presented as one that has been tested against data. In any precise meaning of the concept of hypothesis testing, this implies that other plausible descriptions of the observations have been rejected. By presenting the evidence this way these papers run a great risk of committing a type I statistical error (a false positive). If the present paper had claimed that this hypothesis is false we would have run the same risk of committing a type II error (a false negative). But this is not what we have done here. Our conclusion is that the issue of scaling beyond centennial timescales is still open, in spite of the large body of literature that conveys the impression that it is closed.

\section{The Supplement related to this article is available online at doi:10.5194/esd-7-419-2016-supplement.}

Acknowledgements. This paper was supported by the Norwegian Research Council (KLIMAFORSK programme) under grant no. 229754. We are grateful for discussions with Martin Rypdal and Dmitry Divine, technical assistance from Ola Løvsletten, and Peter Ditlevsen for providing the high-resolution GRIP data set.

Edited by: G. Lohmann

\section{References}

Alley, R. B. and Agustsdottir, A. M.: The 8k event: cause and consequences of a major Holocene abrupt climate change, Quaternary Sci. Rev., 24, 1123-1149, doi:10.1016/j.quascirev.2004.12.004, 2005.

Berner, K. S., Koc, N., Divine, D., Godtliebsen, F., and Moros, M.: A decadal-scale Holocene sea surface temperature record from the subpolar North Aatlantic constructed using diatoms and statistics and its relation to other climate parameters, Paleoceanography, 23, PA2210, doi:10.1029/2006PA001339, 2008.

Blender, R. and Fraedrich, K.: Long time memory in global warming simulations, Geophys. Res. Lett., 30, 1769-1772, doi:10.1029/2003GL017666, 2003.

Blender, R., Fraedrich, K., and Hunt, B.: Millennial climate variability: GCM-simulation and Greenland ice cores, Geophys. Res. Lett., 33, L04710, doi:10.1029/2005GL024919, 2006.

Bond, G., Showers, W., Cheseby, M., Lotti, R., Almasi, P., deMenocal, P., Priore, P., Cullen, H., Hajdas, I., and Bonani, G.: A Pervasive Millennial-Scale Cycle in North Atlantic Holocene and Glacial Climates, Science, 278, 1257-1266, doi:10.1126/science.278.5341.1257, 1997.

Bond, G. C. and Lotti, R.: Iceberg Discharges into the North Atlantic on Millennial Time Scales During the Last Glaciation, Science, 267, 1005-1010, doi:10.1126/science.267.5200.1005, 1995.

Briffa, K. R., Osborn, T. J., Schweingruber, F. H., Harris, I. C., Jones, P. D., Shiyatov, S. G., and Vaganov, E. A.: Lowfrequency temperature variations from a northern tree ring density network, J. Geophys. Res.-Atmos, 106, 2929-2941, doi:10.1029/2000JD900617, 2001.

Brohan, P., Kennedy, J. J., Harris, I., Tett, S. F. B., and Jones, P. D.: Uncertainty estimates in regional and global observed temperature changes: A new data set from 1850, J. Geophys. Res., 111, D12106, doi:10.1029/2005JD006548, 2006.

Dansgaard, W., Johnsen, S. J., Clausen, H. B., Dahl-Jensen, D., Gundestrup, N. S., Hammer, C. U., Hvidberg, C. S., Steffensen, J. P., Sveinbjörnsdottir, A. E., Jouzel, J., and Bond, G.: Evidence for general instability of past climate from a 250-kyr ice-core record, Nature, 364, 218-220, doi:10.1038/364218a0, 1993.

Ditlevsen, P. D., Svensmark, H., and Johnsen, S.: Contrasting atmospheric and climate dynamics of the last-glacial and Holocene periods, Nature, 379, 810-812, doi:10.1038/379810a0, 1996.

Dokken, T. M., Nisancioglu, K. H., Li, C., Battisti, D. S., and Kissel, C.: Dansgaard-Oeschger cycles: Interactions between ocean and sea ice intrinsic to the Nordic seas, Paleoceanography, 28, 491502, doi:10.1002/palo.20042, 2013.

Efstathiou, M. N., Tzanis, C., Cracknell, A. P., and Varotsos, C. A.: New features of land and sea surface temperature anomalies, Int. J. Remote Sens., 32, 3231-3238, doi:10.1080/01431161.2010.541504, 2011. 
EPICA community members: Eight glacial cycles from an Antarctic ice core, Nature, 429, 623-628, doi:10.1038/nature02599, 2004.

Esper, J., Cook, E. R., and Schweingruber, F. H.: Low-Frequency Signals in Long Tree-Ring Chronologies for Reconstructing Past Temperature Variability, Science, 295, 2250-2253, doi:10.1126/science.1066208, 2002.

Fraedrich, K. and Blender, R.: Scaling of Atmosphere and Ocean Temperature Correlations in Observations and Climate Models, Phys. Rev. Lett., 90, 108501, doi:10.1103/PhysRevLett.90.108501, 2003.

Fredriksen, H.-B. and Rypdal, K.: Spectral Characteristics of Instrumental and Climate Model Surface Temperatures, J. Climate, 29, 1253-1268, doi:10.1175/JCLI-D-15-0457.1, 2016.

Geoffroy, O., Saint-Martin, D., Olivié, D. J. L., Voldoire, A., Bellon, G., and Tytéca, S.: Transient Climate Response in a Two-Layer Energy-Balance Model. Part I: Analytical Solution and Parameter Calibration Using CMIP5 AOGCM Experiments, J. Climate, 26, 1841-1857, doi:10.1175/JCLI-D-12-00195.1, 2013.

Greenland Ice Core Project (GRIP) Members: Climate instability during the last interglacial period recorded in the GRIP ice core, Nature, 364, 203-207, doi:10.1038/364203a0, 1993.

Huang, S.: Merging information from different resources for new insights into climate change in the past and future, Geophys. Res. Lett., 31, L13205, doi:10.1029/2004GL019781, 2004.

Huybers, P. and Curry, W.: Links between annual, Milankovitch and continuum temperature variability, Nature, 441, 329-332, doi:10.1038/nature04745, 2006.

Johnsen, S. J., Clausen, H. B., Dansgaard, W., Gundestrup, N. S., Hammer, C. U., Andersden, U., Andersen, K. K., Hvidberg, C. S., Dahl-Jensen, D., Steffensen, J. P., Shoji, H., Sveinbjörnsdottir, A. E., White, J., Jouzel, J., and Fisher, D.: The ${ }^{18} \mathrm{O}$ record along the Greenland Ice Core Project deep ice core and the problem of possible Eemian climatic instability, J. Geophys. Res.Oceans, 102, 26397-26410, doi:10.1029/97JC00167, 1997.

Jones, P. D., Briffa, K. R., Barnett, T. P., and Tett, S. F. B.: Highresolution palaeoclimatic records for the last millennium: interpretation, integration and comparison with General Circulation Model control-run temperatures, Holocene, 8, 455-471, doi:10.1191/095968398667194956, 1998.

Jouzel, J., Masson-Delmotte, V., Cattani, O., Dreyfus, G., Falourd, S., Hoffmann, G., Minster, B., Nouet, J., Barnola, J. M., Chappellaz, J., Fischer, H., Gallet, J. C., Johnsen, S., Leuenberger, M., Loulergue, L., Luethi, D., Oerter, H., Parrenin, F., Raisbeck, G., Raynaud, D., Schilt, A., Schwander, J., Selmo, E., Souchez, R., Spahni, R., Stauffer, B., Steffensen, J. P., Stenni, B., Stocker, T. F., Tison, J. L., Werner, M., and Wolff, E. W.: Orbital and Millennial Antarctic Climate Variability over the Past 800,000 Years, Science, 317, 793-796, doi:10.1126/science.1141038, 2007.

Kantelhardt, J. W.: Fractal and Multifractal Time Series, in: Mathematics of Complexity and Dynamical Systems, edited by: Meyers, R. A., Springer New York, 463-487, doi:10.1007/978-14614-1806-1_30, 2011.

Kantelhardt, J. W., Koscielny-Bunde, E., Rego, H. H. A., Havlin, S., and Bunde, A.: Detecting long-range correlations with detrended fluctuation analysis, Physica A, 295, 441-454, doi:10.1016/s0378-4371(01)00144-3, 2001.

Koscielny-Bunde, A. B., Havlin, S., and Goldreich, Y.: Analysis of daily temperature fluctuations, Physica A, 231, 393-396, doi:10.1016/0378-4371(96)00187-2, 1996.
Laepple, T. and Huybers, P.: Ocean surface temperature variability: Large model-data differences at decadal and longer periods, P. Natl. Acad. Sci. USA, 111, 16682-16687, doi:10.1073/pnas.1412077111, 2014.

Lomb, N. R.: Least-squares frequency analysis of unequally spaced data, Astrophys. Space Sci., 39, 447-462, 1976.

Lovejoy, S. and Schertzer, D.: Haar wavelets, fluctuations and structure functions: convenient choices for geophysics, Nonlin. Processes Geophys., 19, 513-527, doi:10.5194/npg-19-513-2012, 2012a.

Lovejoy, S. and Schertzer, D.: Low Frequency Weather and the Emergence of the Climate, American Geophysical Union, Washington D.C., 231-254, doi:10.1029/2011GM001087, 2012 b.

Lovejoy, S. and Schertzer, D.: The Weather and Climate: Emergent Laws and Multifractal Cascades, Cambridge University Press, New York, NY, 383-405, 2013.

Lovejoy, S., Schertzer, D., and Varon, D.: Do GCMs predict the climate ... or macroweather?, Earth Syst. Dynam., 4, 439-454, doi:10.5194/esd-4-439-2013, 2013.

Lovejoy, S., del Rio Amador, L., and Hébert, R.: The ScaLIng Macroweather Model (SLIMM): using scaling to forecast globalscale macroweather from months to decades, Earth Syst. Dynam., 6, 637-658, doi:10.5194/esd-6-637-2015, 2015.

Mann, M. E.: On long range dependence in global surface temperature series, Climatic Change, 107, 267-276, doi:10.1007/s10584-010-9998-z, 2011.

Mann, M. E., Bradley, R. S., and Hughes, M. K.: Northern Hemisphere temperatures during the past millennium: Inferences, uncertainties, and limitations, Geophys. Res. Lett., 26, 759-762, doi:10.1029/1999GL900070, 1999.

Mann, M. E., Zhang, Z., Hughes, M. K., Bradley, R. S., Miller, S. K., Rutherford, S. and Ni, F.: Proxy-based reconstructions of hemispheric and global surface temperature variations over the past two millennia, P. Natl. Acad. Sci. USA, 105, 13252, doi:10.1073/pnas.0805721105, 2008.

Marcott, S. A. a. D. S., Clark, P. U., and Mix, A. C.: A Reconstruction of Regional and Global Temperature for the Past 11,300 Years, Science, 339, 1198-1201, doi:10.1126/science.1228026, 2013.

Masson, V., Vimeux, F., Jouzel, J., Morgan, V., Delmotte, M., Ciais, P., Hammer, C., Johnsen, S., Lipenkov, V. Y., MosleyThompson, E., Petit, J.-R., Steig, E. J., Stievenard, M., and Vaikmae, R.: Holocene Climate Variability in Antarctica Based on 11 Ice-Core Isotopic Records, Quaternary Res., 54, 348-358, doi:10.1006/qres.2000.2172, 2000.

Moberg, A., Sonechkin, D. M., Holmgren, K., Datsenko, N. M., and Karlén, W.: Highly variable Northern Hemisphere temperatures reconstructed from low-and high-resolution proxy data, Nature, 433, 613-617, doi:10.1038/nature03265, 2005.

Neukom, R., Gergis, J., Karoly, D. J., Wanner, H., Curran, M., Elbert, J., Gonzalez-Rouco, F., Linsley, B. K., Moy, A. D., Mundo, I., Raible, C. C., Steig, E. J., van Ommen, T., Vance, T., Villalba, R., Zinke, J., and Frank, D.: Inter-hemispheric temperature variability over the past millennium, Nat. Clim. Change, 4, 362-367, doi:10.1038/nclimate2174, 2014.

Østvand, L., Nilsen, T., Rypdal, K., Divine, D., and Rypdal, M.: Long-range memory in internal and forced dynamics of millenium-long climate model simulations, Earth Syst. Dynam., 5, 295-308, doi:10.5194/esd-5-295-2014, 2014. 
Pelletier, J. D.: The power spectral density of atmosphereic temperature from time scales of $10^{-2}$ to $10^{6} \mathrm{yr}$, Earth Planet. Sc. Lett., 158, 157-164, doi:10.1016/S0012-821X(98)00051-X, 1998.

Peng, C.-K., Buldyrev, S. V., Havlin, S., Simons, M., Stanley, H. E., and Goldberger, A. L.: Mosaic organization of DNA, Phys. Rev. E, 49, 1685-1689, doi:10.1103/PhysRevE.49.1685, 1994.

Rehfeld, K., Marwan, N., Heitzig, J., and Kurths, J.: Comparison of correlation analysis techniques for irregularly sampled time series, Nonlin. Processes Geophys., 18, 389-404, doi:10.5194/npg18-389-2011, 2011.

Roe, G. H. and Steig, E. J.: Characterization of Millennial-scale Climate Variability, J. Climate, 17, 1929-1944, doi:10.1175/15200442(2004)017<1929:COMCV>2.0.CO;2, 2004.

Rybski, D., Bunde, A., Havlin, S., and von Storch, H.: Long-term persistence in climate and the detection problem, Geophys. Res. Lett., 33, L06718, doi:10.1029/2005GL025591, 2006.

Rypdal, K.: Global temperature response to radiative forcing: Solar cycle versus volcanic eruptions, J. Geophys. Res., 117, D06115, doi:10.1029/2011JA017283, 2012.

Rypdal, K.: Attribution in the presence of a long-memory climate response, Earth Syst. Dynam., 6, 719-730, doi:10.5194/esd-6719-2015, 2015.

Rypdal, M.: Early-Warning Signals for the onsets of Greenland Interstadials and the Younger Dryas-Preboreal transition, J. Climate, doi:10.1175/JCLI-D-15-0828.1, in press, 2016.

Rypdal, M. and Rypdal, K.: Testing Hypotheses about SunClimate Complexity Linking, Phys. Rev. Lett., 104, 128501, doi:10.1103/PhysRevLett.104.128501, 2010.
Rypdal, M. and Rypdal, K.: Long-memory effects in linearresponse models of Earth's temperature and implications for future global warming, J. Climate, 27, 5240-5258, doi:10.1175/JCLI-D-13-00296.1, 2014.

Rypdal, M. and Rypdal, K.: Late Quaternary temperature variability described as abrupt transitions on a $1 / f$ noise background, Earth Syst. Dynam., 7, 281-293, doi:10.5194/esd-7-281-2016, 2016.

Rypdal, K., Østvand, L., and Rypdal, M.: Long-range memory in Earth's surface temperature on time scales from months to centuries, J. Geophys. Res., 118, 7046-7062, doi:10.1002/jgrd.50399, 2013.

Rypdal, K., Rypdal, M., and Fredriksen, H.-B.: Spatiotemporal Long-Range Persistence in Earth's Temperature Field: Analysis of Stochastic-Diffusive Energy Balance Models, J. Climate, 28, 8379-8395, doi:10.1175/JCLI-D-15-0183.1, 2015.

Scargle, J. D.: Studies in astronomical time series analysis. Statistical aspects of spectral analysis of unevenly sampled data, Astrophys. J., 263, 835-853, doi:10.1086/160554, 1982.

Torrence, C. and Compo, G. P.: A Practical Guide to Wavelet Analysis, B. Am. Meteorol. Soc., 79, 61-78, doi:10.1175/15200477(1998)079<0061:APGTWA>2.0.CO;2, 1998.

WAIS Divide Project Members: Precise interpolar phasing of abrupt climate change during the last ice age, Nature, 520, 661-665, doi:10.1038/nature14401, 2015. 